A new subfamily of fossorial colubroid snakes from the Western Ghats of peninsular India

V. Deepak ${ }^{\mathrm{a}, \mathrm{b}^{*}}$, Sara Ruane ${ }^{\mathrm{c}}$, David J. Gower ${ }^{\mathrm{a}}$

${ }^{a}$ Department of Life Sciences, The Natural History Museum, London SW7 5BD, UK;

${ }^{b}$ Centre for Ecological Sciences, Indian Institute of Science, Bangalore, 560012,

India; ${ }^{c}$ Department of Biological Sciences, Rutgers University-Newark, 195

University Ave, Newark, New Jersey, 07102, USA; ${ }^{*}$ Corresponding author

${ }^{a}$ Department of Life Sciences, The Natural History Museum, London SW7 5BD, UK.

E-mail: veerappandeepak@gmail.com 


\title{
A new subfamily of fossorial colubroid snakes from the Western Ghats of peninsular India
}

\begin{abstract}
We report molecular phylogenetic and dating analyses of snakes that include new mitochondrial and nuclear DNA sequence data for three species of the peninsular Indian endemic Xylophis. The results provide the first molecular genetic test of and support for the monophyly of Xylophis. Our phylogenetic results support the findings of a previous, taxonomically restricted phylogenomic analysis of ultraconserved nuclear sequences in recovering the fossorial Xylophis as the sister taxon of a clade comprising all three recognised extant genera of the molluscivoran and typically arboreal pareids. The split between Xylophis and 'pareids' is estimated to have occurred on a similar timescale to that between most (sub)families of extant snakes. Based on phylogenetic relationships, depth of molecular genetic and estimated temporal divergence, and on the external morphological and ecological distinctiveness of the two lineages, we classify Xylophis in a newly erected subfamily (Xylophiinae subfam. nov.) within Pareidae.
\end{abstract}

\section{Key words}

Asia, classification, Pareidae, Pareinae, phylogenetics, Xylophis, taxonomy

\section{Introduction}

The caenophidian snake genus Xylophis Beddome, 1878 contains three currently recognised species of small fossorial snakes endemic to the southern part of the 
Western Ghats of peninsular India (Gower and Winkler 2007, Wallach et al. 2014;

Srinivasulu et al. 2014). Morphological systematists have not settled on the phylogenetic relationships of Xylophis or of its corresponding suprageneric classification. For example, Underwood (1967) included Xylophis in his concept of Dipsadidae, a group comprising xenodermines, pareines, calamarines, sibynophiines, lycodontines, xenodontines and some then enigmatic Asian natricines (including the Sri Lankan Aspidura - to which at least superficial similarities to Xylophis were noted by Gans and Fetcho 1982, Dowling and Pinou 2003, Gower and Winkler 2007, and Simões et al. 2016). Since Underwood's (1967) work, Xylophis has been considered to be a xenodermid (or xenodermatid/xenodematine/xenodermine depending on authority) (McDowell 1987, 2008, Wallach 1998, Zaher 1999, Vidal 2002, Lawson et al. 2005, Cundall and Irish 2008: 556, 573, Zaher et al. 2009, Pyron et al. 2013, Dowling and Pinou 2003, see also Underwood 1967: 98), an elapoid incertae sedis (Wallach et al. 2014), and a colubrine (Cundall and Irish 2008: 645).

Until recently, there were no molecular systematic data available for Xylophis. Simões et al. (2016) published sequences of three visual opsin genes for X. captaini Although noted as not being neutral phylogenetic markers, Simões et al. (2016) reported various phylogenetic results for $X$. captaini for each of these three genes in isolation: for locus $r h 1, X$. captaini was recovered as sister to the pareid Pareas monticola, with this clade being sister to all other sampled non-viperid colubroids; for locus sws $1, X$. captaini, was recovered as sister to all other sampled colubroids $(P$. monticola was not sampled for this gene); for locus $l w s, X$. captaini was recovered as sister to Amphiesma stolata within natricine colubrids (again, P. monticola was not sampled for this gene). Although the sister relationships with P. monticola (for $r h 1$ ) 
and with $A$. stolata (for $l w s$ ) were well supported, most of the deeper internal branches throughout these trees were not well-resolved.

Ruane and Austin (2017) sampled one historical museum specimen of Xylophis stenorhynchus in an application of ultraconserved element loci in snake phylogenomics, combining their historical sampling with modern snake sample data from Streicher and Wiens (2016). Ruane and Austin's sampling was sparse (17 species of caenophidians, including one xenodermid and no natricines) but $X$. stenorhynchus was recovered as the well-supported sister taxon to the single sampled pareid, Pareas hamptoni, and the number of ultraconserved elements generated for $X$. stenorhynchus (2546 loci) was on par with modern samples (see Table 1, Ruane and Austin 2017). As currently conceived, pareids comprise ca. 20 nocturnal, molluscivorous, non-fossorial species (classified in three genera: Pareas Wagler, 1830; Aplopeltura Duméril, 1853; Asthenodipsas Peters, 1864) restricted to east and Southeast Asia, with two species (P. monticola (Cantor, 1839) and $P$. margaritophorus (Jan, 1866)) extending into northeast India (Whitaker and Captain 2004, Uetz et al. 2017). Commenting on their somewhat unexpected phylogenetic result for Xylophis, Ruane and Austin (2017: 5) suggested that the phylogenetic relationships of this genus could be investigated more thoroughly by analysing a wider sample of snakes, including more species of Xylophis.

Here we report sequence data for 'standard' mitochondrial (mt) and nuclear (nu) phylogenetic markers for snakes for three species of Xylophis and include them in broadly taxonomically sampled phylogenetic analyses of extant snakes. These analyses provide the first molecular test of the monophyly of the genus, and the results support classification of Xylophis in a newly erected subfamily within Pareidae. 


\section{Material and methods}

\section{Classification and institutional abbreviations}

We followed the family and subfamily classification used by Uetz et al. (2018), including the recently described subfamilies Ahaetuliinae Figueroa et al. 2016 (within Colubridae) and Cyclocorinae Weinell and Brown, 2018 (within Lamprophiidae). Xylophis tissues were sampled from vouchers deposited in the Bombay Natural History Society, Mumbai, India (BNHS), California Academy of Sciences, San Francisco, USA (CAS), and Centre for Ecological Sciences, IISc, Bengaluru, India (CES).

\section{Molecular data and phylogenetic analysis}

We generated DNA sequence data for two specimens from freshly collected tissue, a Xylophis perroteti from the Nilgiris (CESG 2016b) and a X. captaini from the type locality Kannam, Kottayam District (BNHS 3376). Genomic DNA was extracted from liver tissue samples stored in absolute ethanol at $-20^{\circ} \mathrm{C}$. DNeasy (Qiagen ${ }^{\mathrm{TM}}$ ) blood and tissue kits were used to extract DNA. We amplified partial sequences of three mitochondrial (mt) genes and three nuclear $(\mathrm{nu})$ genes. The $\mathrm{mt}$ genes are $16 \mathrm{~S}$ rRNA (16s), cytochrome b (cytb) and NADH dehydrogenase subunit 4 (nd4), and the nu markers are the recombination activating gene 1 ( $\mathrm{rag} l$ ), oocyte maturation factor (cmos), and brain-derived neurotrophic factor ( $b d n f)$. DNA PCR amplification and Sanger sequencing used previously reported primers (Palumbi et al. 1991; Arévalo et al. 1994; Palumbi 1996; Parkinson et al. 2000; Noonan and Chippindale, 2006; Wiens et al. 2008).

We attempted to extract homologous sequences for our phylogenetic markers from the unfiltered, unassembled, raw sequence reads that were generated during 
targeted sequencing of ultra-conserved elements (UCEs) for a historical specimen (CAS 17199) of Xylophis stenorhynchus from Ruane and Austin (2017). These data comprised 32,236,948 reads each for read 1 and read 2. Although none of the loci used in this study was targeted by the UCE probe kit (MYbaits tetrapod 5K kit, which targets 5060 UCEs from amniotes: Faircloth et al. 2012) used by Ruane and Austin (2017), we considered it possible that the loci of interest were sequenced as "bycatch" during the high-throughput sequencing, particularly for mtDNA genes due to the high number of copies of these loci in genomic DNA.

Using the program Geneious (Kearse et al. 2012) Ruane and Austin's (2017) unfiltered reads for $X$. stenorhynchus were mapped to each of the newly generated $X$. captaini and $X$. perroteti Sanger sequences for $16 s, c y t b, n d 4, c m o s, r a g 1$, and $b d n f$ (see Table 1). This was done using the Geneious align/assemble option "map to reference", with the modern sample serving as the reference for the unfiltered $X$. stenorhynchus reads; sensitivity was set to medium-low with up to five iterations. Where successful, the resulting mapped reads of $X$. stenorhynchus were combined into consensus sequences for each marker to be included in subsequent analyses.

We constructed a molecular dataset for 507 leaves $(500$ snakes +7 non-snake squamates; $493+7$ species, respectively) including 14 of the 20 currently recognised species of pareids. Data coverage for each of the genes in the dataset are as follows: cytb $80.9 \%$, 16 s $68.1 \%$, nd4 $58.8 \%$, cmos $71.6 \%$, bdnf $30.4 \%$ and ragl $13.6 \%$. GenBank accession numbers for all sequences included in our phylogenetic and dating analyses are presented in Table S1. Alignments per gene were carried out in MEGA 5 (Tamura et al. 2011) using the ClustalW algorithm with default parameters and are available online from the Natural History Museum data portal (http://data.nhm.ac.uk/ dataset/deepak-xylophis). Uncorrected p-distances and Kimura 
2-parameter (Kimura, 1980) distances were calculated using MEGA 5. Phylogenetic analysis was implemented in the program RAxML v7.4.2 GUI (Stamatakis, 2006; Silvestro and Michalak 2012) with the six gene concatenated dataset. This dataset was 4,557bp long and was partitioned by gene and by codon, a total of 11 partitions (see Table S2), determined as the best-fit scheme using ParitionFinder 1.2 (Lanfear et al. 2012). We used GTRGAMMA model in RAxML which is recommended over the GTR $+\mathrm{G}+\mathrm{I}$ because the 25 rate categories account for potentially invariant sites (Stamatakis, 2006), as was also implemented in other large-scale snake molecular phylogenetic analyses (Pyron et al. 2011; Zaher et al. 2012; Figueroa et al. 2016).

Divergence times were estimated using a subset of taxa for the same genes, with a dataset containing 81 snake species including representatives of all extant subfamilies of alethinophidian snakes and all extant families of scolecophidians (Table 1). These data were newly aligned (using the methods outlined above, alignment available at: http://data.nhm.ac.uk/ dataset/deepak-xylophis), producing a dataset 4,504bp. PartitionFinder 1.2 (Lanfear et al. 2012) was used to identify the best-fitting partition scheme and model(s) of sequence evolution according to the Bayesian Information Criterion (BIC) using the default greedy algorithm with linked branch lengths (see Table S3 for partitions and models). We explored the sensitivity of our phylogenetic results to our selected (ClustalW) alignment method by alternatively aligning the $16 \mathrm{~s}$ data also with MUSCLE (Edgar, 2004), as well as using Gblocks v0.91b (Castresana, 2000) to identify and remove ambiguously aligned sites from the ClustalW alignment using the 'less stringent' option. These alternative approaches to the $16 \mathrm{~s}$ data did not notably change the topology or support values in optimal RAxML (data partitioned by gene and by codon) trees for the concatenated data (Fig. S1). 
Divergence times were estimated using a Bayesian relaxed uncorrelated lognormal clock model implemented in BEAST 1.8.2 (Drummond et al. 2012). We used fossil calibrations recommended by Head (2015) and Head et al. (2016) to date minimum ages of five divergences: (1) oldest divergence within crown Alethinophidia based on Haasiophis terrasanctus Tchernov et al. 2000; minimum age 93.9 Ma, soft maximum 100.5 Ma, (2) oldest divergence between non-xenodermid colubroids and their closest living relative (Xenodermidae in our tree), based on Procerophis sahnii Rage et al. 2008; minimum 50.5 Ma, soft maximum 72.1 Ma, (3) divergence between Boinae and its sister taxon (Erycinae + Candoiinae in our tree) based on Titanoboa cerrejonensis Head et al. 2009; minimum 58 Ma, soft maximum $64 \mathrm{Ma}$, and (4) divergence between Viperinae and Crotalinae based on Vipera aspis complex (Szyndlar and Rage 1999); minimum 20.0 Ma, soft maximum 23.8 Ma, and (5) oldest divergence within elapids based on Naja romani (Hoffstetter, 1939); minimum age $17 \mathrm{Ma}$, soft maximum $60 \mathrm{Ma}$ (see Table S4 for exact values applied to each calibration prior). Analyses used random starting trees, with clock and tree models linked across partitions. Two independent analyses were run for $600,000,000$ generations sampling every 5000 trees, the effective sample size (ESS) values were evaluated using Tracer 1.6 (Rambaut et al. 2014). The prior distribution for all fossil calibrations was set to lognormal.

\section{Results}

\section{Phylogenetic inference}

Mapping the Sanger sequencing data for Xylophis captaini and X. perroteti against the unfiltered $X$. stenorhynchus high-throughput sequence reads resulted in potentially homologous consensus sequences for the latter for $16 s$ (441bp; 103 reads assembled), 
cytb (513bp; 60 reads), $n d 4$ (328bp; 61 reads), cmos (169bp; 6 reads), and bdnf (91bp; 4 reads). These consensus sequences are reported in Table 1 . These sequences were similar to those of $X$. perroteti (uncorrected p-distances 0.078 for $16 s ; 0.204$ for $n d 4$; 0.037 for cmos; 0.045 for $b d n f$ ) and $X$. captaini (uncorrected p-distances 0.114 for $16 s ; 0.221$ for $c y t b)$.

Our ML phylogenetic analysis provides strong support for the monophyly of Xylophis and for the sister group relationship between Xylophis and a clade comprising the pareids Pareas, Aplopeltura and Asthenodipsas (Figs. 1 and S2). Within the latter clade, Pareas and Asthenodipsas are strongly supported as monophyletic, with the former being sister to the monotypic Aplopeltura. The Xylophis, Pareas, Aplopeltura and Asthenodipsas clade (here considered to comprise Pareidae) is recovered as a member of a lineage comprising all colubroids except Xenodermidae. Although there is strong signal for pareids lying outside a group comprising most other colubroids, the relationships among Pareidae, Viperidae and all other non-xenodermid colubroids are not clearly resolved by our analyses. Uncorrected p-distances between Xylophis and the other three pareid genera for the sampled genes are $0.07-0.12(16 s), 0.27-0.38(c y t b), 0.21-0.25(n d 4), 0.03-0.1$ (cmos) and 0.03 (bdnf). Pairwise distances between recognised colubroid families and between intrafamilial subfamilies for cmos and $b d n f$ (reported in Tables S5-S8) are summarised in Fig. 2. The molecular dating analysis recovers an estimated minimum divergence of 55-35 Ma between Xylophis and its sister taxon (Pareas, Aplopeltura and Asthenodipsas) (Fig. 3). Rerunning the dating analysis but excluding third codon positions of the mitochondrial genes $c y t b$ and $n d 4$ did not notably alter the results for most divergences (including that for Xylophis versus its sister) in terms of relative 
ages (Fig. S3), with estimated divergence dates for the two analytical treatments being strongly correlated (Fig. S4).

\section{Systematics}

Based on the well-supported inferred phylogenetic relationships of Xylophis and divergence from its extant sister lineage, we refer the genus to the family Pareidae, and re-define the latter phylogenetically as all snakes more closely related to Pareas carinatus Wagler, 1830 than to Xenodermus javanicus Rheinhardt, 1836, Vipera aspis (Linnaeus, 1758) or Homalopsis buccatus (Linnaeus, 1758). Given the molecular genetic and phenotypic distinctiveness of the two lineages comprising the basal split within Pareidae, we classify Pareas, Aplopeltura and Asthenodipsas within the subfamily Pareinae (defined phylogenetically as all snakes more closely related to Pareas carinatus Wagler, 1830 than to Xylophis perroteti Duméril, Bibron and Duméril, 1854) and we erect a new subfamily for Xylophis:

DIAPSIDA Osborn, 1903

Superorder LEPIDOSAURIA Haeckel, 1866

Order SQUAMATA Oppel, 1811

Suborder SERPENTES Linnaeus, 1758

Infraorder CAENOPHIDIA Hoffstetter, 1939

Superfamily COLUBROIDEA Oppel, 1811

Family PAREIDAE Romer, 1956

Subfamily Xylophiinae subfam. nov.

\section{Type genus}


Xylophis Beddome, 1878

\section{Content}

A single genus with three currently recognised species: $X$. stenorhynchus (Günther, 1875); X. perroteti Duméril, Bibron and Duméril, 1854; X. captaini Gower and Winkler, 2007. Xylophis indicus Beddome, 1878 has been considered a synonym of X. stenorhynchus (e.g., Smith 1943, Wallach et al. 2014) but might also be valid (Gower and Winkler 2007). Xylophis perroteti includes the synonyms Rhadosoma microcephalum Günther, 1858 (e.g., Smith 1943, Wallach et al. 2014).

\section{Phylogenetic definition}

All snakes more closely related to Xylophis perroteti than to Pareas carinatus Wagler, 1830.

\section{Diagnosis}

Colubroid snakes with first (anteriormost) three pairs of infralabial shields reduced to narrow strips, together much smaller than large pair of anterior chin (genial) shields.

\section{Distribution}

The Western Ghats region of peninsular India. Xylophis is thus far known only from the southern part of the Western Ghats, in the states of Kerala and Tamil Nadu (Fig. 4). Species of the genus have been recorded from close to sea level (Xylophis captaini: Gower and Winkler 2007) to at least 2,000 m (X. perroteti: Srinivasulu et al. 2014).

\section{Discussion}


Age of divergence (whether absolute or relative) between sister lineages has sometimes been applied as a secondary criterion in recognition of suprageneric taxa (e.g., Wilkinson et al. 2011), including the formal naming of new families (e.g. Kamei et al. 2012). Although the general application of such a criterion has been cautioned against (e.g., Vences et al. 2013; Frost 2017: see "comments on taxonomy related to version 5.6"), we see some merit in using estimated divergence age cautiously as an additional guide alongside phylogenetic relationships and extent of phenotypic and raw molecular genetic divergence. In this case, we take some comfort in naming a new subfamily given that the estimated age of divergence of Xylophiinae from Pareinae is comparable to that between sister pairs of other snake (sub)families (Figs. 3 and S3).

Although xylophiines and pareines are phenotypically disparate superficially, the anatomy and anatomical diversity of these two lineages (and of other major lineages of colubroids) is insufficiently known to yet rule out the identification of unambiguous synapomorphies for Pareidae. Although classifying Xylophis as a xenodermine on the basis of skull, head muscle and hemipenis features, McDowell (1987: 35-36) also drew attention between at least $X$. perroteti and pareines (and calamariines) in terms of posteriorly extensive kidneys and a distinct rectal caecum. The morphology of Xylophis is poorly studied and further work in the light of the renewed interest in its phylogenetic relationships seems warranted.

The evolutionary divergence between xylophiines and pareines resulted in sister clades with markedly differing distributions, morphologies and ecologies. Although both lineages comprise small to moderately sized predators of invertebrates, xylophiines are small-headed, small-eyed, fossorial, relative generalist or opportunistic predators (Kumar and Kannan 2017) restricted to the southern part of 
the Western Ghats of peninsular India, while pareines are relatively larger-headed (head greater in girth than anterior of body), large-eyed, surface dwelling (often arboreal) specialist molluscivores (Cundall and Greene, 2000) restricted almost entirely to east and southeast Asia (also extending into northeast India; Fig. 4). Given that the Indian subcontinent (part of Gondwana) did not accrete with the rest of Asia until ca.55 Ma (Patriat and Achache, 1984), our estimated divergence between xylophiines and pareines $(55-35 \mathrm{Ma})$ is consistent with dispersal of either a peninsular Indian ancestor into east/southeast Asia or vice versa. This hypothesis is a little more parsimonious than one invoking a widespread ancestral pareid lineage with followed by spatially exclusive extinctions of xylophiines (in east and southeast Asia) and pareines (in peninsular India). However, that Pareidae, Xenodermidae and Acrochordidae are all Asian and that they might comprise a paraphyletic assemblage lying successively outside of a clade (= Endoglyptodonta of Zaher et al. 2009) comprising Viperidae, Elapidae, Colubridae, and Lamprophiidae (e.g. Vidal et al. 2007, Zaher et al. 2009, Grazziotin et al. 2012, Pyron et al. 2013, Figueroa et al. 2016) is more supportive of an Asian (rather than Gondwanan) origin of Pareidae, and thus of a dispersal of the ancestor of the Xylophiinae lineage into peninsular India from east or southeast Asia rather than vice versa. Resolution of the phylogenetic position of the northeast Indian Pareas moniticola and P. margaritophorus might usefully inform the question of the historical biogeography of Pareidae (or at least of Pareinae).

\section{Geolocation Information}

Study Area (box): $8.65000^{\circ} \mathrm{N}, 76.95000^{\circ} \mathrm{E}$ to $11.31198^{\circ} \mathrm{N}, 76.58653^{\circ} \mathrm{E}$

\section{Acknowledgements}


In addition to people acknowledged by Gower and Winkler (2007), we thank Srihari Ananthakrishna and Krishna Chaitanya for their support during fieldwork. Chinta Sidharthan is thanked for help with some of the lab work, and Mark Wilkinson and Natalie Cooper for help with some of the analyses. VD thanks Achyuthan Srikanthan for sharing Xylophis perroteti photos. We also further thank those acknowledged in Ruane and Austin (2017), especially Jens Vindum (California Academy of Sciences) for the loan of Xylophis stenorhynchus and Jeff Streicher for sharing the dataset from Streicher and Wiens (2016). Part of this project was made possible from NSF DEB1146033 to C.C. Austin. VD's contribution was support, in part, by Marie Skłodowska-Curie Fellowship EU project 751567.

\section{References}

Arévalo E, Davis SK, Sites JW. 1994. Mitochondrial DNA sequence divergence and phylogenetic relationships among eight chromosome races of Sceloporus grammicus complex (Phrynosomatidae) in Central Mexico. Syst Biol. 43: $387-418$.

Castresana J. 2000. Selection of conserved blocks from multiple alignments for their use in phylogenetic analysis. Mol Biol Evol. 17: 540-552.

Cundall D, Irish FJ. 2008. The snake skull. In: Gans C, Gaunt AS, Adler K, editors. Biology of the Reptilia, v.20 (Morphology H: The Skull of Lepidosauria). Ithaca, New York: Society for the Study of Amphibians and Reptiles; p. 349692.

Cundall D, Greene HW. 2000. Feeding in snakes. In: Schwenk K, editor. Feeding form, function, and evolution in tetrapod vertebrates. San Diego, CA: Academic Press; p. 293-333. 
Dowling HG, Pinou T. 2003. Xenodermatid snakes in America. Herpetol Rev. 34: $20-23$.

Drummond AJ, Suchard MA, Xie D, Rambaut A. 2012. Bayesian phylogenetics with BEAUti and the BEAST 1.7. Mol Biol Evol. 29: 1969-1973.

Edgar RC. 2004. MUSCLE: multiple sequence alignment with high accuracy and high throughput. Nucleic Acids Res. 32: 1792-1797.

Faircloth BC, McCormack JE, Crawford NG, Harvey MG, Brumfield RT, Glenn TC. 2012. Ultraconserved elements anchor thousands of genetic markers spanning multiple evolutionary timescales. Sys Biol. 61: 717-726.

Figueroa A, McKelvy AD, Grismer LL, Bell CD, Lailvaux SP. 2016. A species-level phylogeny of extant snakes with description of a new colubrid subfamily and genus. PLoS One, 11(9), p.e0161070.

Frost DR. 2017. Amphibian Species of the World: an Online Reference. Version 6.0 (Date of access). Electronic Database accessible at http://research.amnh.org/herpetology/amphibia/index.html. American Museum of Natural History, New York, USA

Gans C, Fetcho JR. 1982. The Sri Lankan genus Aspidura (Serpentes, Reptilia, Colubridae). Carnegie Mus Nat Hist. 51: 271-316.

Gower DJ, Winkler JD. 2007. Taxonomy of the Indian snake Xylophis Beddome (Serpentes: Caenophidia), with description of a new species. Hamadryad 31: $315-329$ 
Grazziotin FG, Zaher H, Murphy RW, Scrocchi G, Benavides MA, Zhang YP, Bonatto SL. 2012. Molecular phylogeny of the new world Dipsadidae (Serpentes: Colubroidea): a reappraisal. Cladistics 28: 437-459.

Günther A. 1858. Catalogue of Colubrine snakes of the British Museum. London.

Head JJ, Mahlow K, Müller J. 2016. Fossil calibration dates for molecular phylogenetic analysis of snakes 2: Caenophidia, Colubroidea, Elapoidea, Colubridae. Palaeontol Electron. 19: 1-21.

Head JJ. 2015. Fossil calibration dates for molecular phylogenetic analysis of snakes 1: Serpentes, Alethinophidia, Boidae, Pythonidae. Palaeontol Electron 18: 117.

Kamei RG, San Mauro D, Gower DJ, Van Bocxlaer I, Sherratt E, Thomas A, Babu S, Bossuyt F, Wilkinson M, Biju SD. 2012. Discovery of a new family of amphibians from northeast India with ancient links to Africa. Proc R Soc Lond [Biol]. 279: 2396-2401.

Kearse M, Moir R, Wilson A, Stones-Havas S, Cheung M, Sturrock S, Buxton S, Cooper A, Markowitz S, Duran C, Thierer T. 2012. Geneious Basic: an integrated and extendable desktop software platform for the organization and analysis of sequence data. Bioinformatics 28: 1647-1649.

Kimura M. 1980. A simple method for estimating evolutionary rate of base substitutions through comparative studies of nucleotide sequences. J Mol Evol $16: 111-120$.

Kumar PS, Kannan P. 2017. Striped Narrow-headed Snake: Diet of the endemic Xylophis perroteti in Nilgiris. Reptile Rap 32: 22-23. 
Lanfear R, Calcott B, Ho SYW, Guindon S. 2012. Partitionfinder: combined selection of partitioning schemes and substitution models for phylogenetic analyses. Mol Biol Evol. 29: 1695-1701.

Lawson R, Slowinski JB, Crother BI, Burbrink FT. 2005. Phylogeny of the Colubroidea (Serpentes): new evidence from mitochondrial and nuclear genes. Mol Phylogenet Evol. 37: 581-601.

McDowell SB, 1987. Systematics. In: Seigel RA, Collins JT, Novak SS, editors. Snakes: Ecology and evolutionary biology. New York: Macmillan Publishing; p. $3-50$.

Noonan BP, Chippindale PT. 2006. Dispersal and vicariance: the complex evolutionary history of boid snakes. Mol Phylogenet Evol. 40: 347-358.

Palumbi SR, Martin AP, Romano SL, McMillan WO, Stice L, Grabowski G. 1991. The Simple Fool's Guide to PCR ver. 2. University of Hawaii, Honolulu.

Palumbi SR. 1996. The polymerase chain reaction. In: Hillis DM, Moritz C, Mable BK, editors. Molecular systematics. 2nd Edition. Massachusetts: Sinauer Associates; p. 205-247.

Parkinson CL, Zamudio KR, Greene HW. 2000. Phylogeography of the pitviper clade Agkistrodon: historical ecology, species status, and conservation of cantils. Mol Ecol. 9: 411-420.

Patriat P, Achache J. 1984. India-Asia collision chronology has implications for crustal shortening and driving mechanism of plates. Nature 311: 615-621. 
Pyron RA, Burbrink FT, Wiens JJ. 2013. A phylogeny and revised classification of Squamata, including 4161 species of lizards and snakes. BMC Evol Biol 13: 93.

Pyron RA, Burbrink FT, Colli GR, de Oca ANM, Vitt LJ, Kuczynski CA, Wiens JJ. 2011. The phylogeny of advanced snakes (Colubroidea), with discovery of a new subfamily and comparison of support methods for likelihood trees. Mol Phylogenet Evol. 58: 329-342.

Rambaut A, Suchard MA, Xie D, Drummond AJ. 2014. Tracer v1. 6. Available from http://beast.bio.ed.ac.uk.Tracer (accessed July 27, 2014).

Ruane S, Austin CC. 2017. Phylogenomics using formalin-fixed and 100+ year-old intractable natural history specimens. Mol Ecol Resour. 17: 1003-1008.

Savage JM. 2015. What are the correct family names for the taxa that include the snake genera Xenodermus, Pareas, and Calamaria? Herpetol Rev. 46: 664665.

Simões BF, Sampaio FL, Douglas RH, Kodandaramaiah U, Casewell NR, Harrison RA, Hart NS, Partridge JC, Hunt DM, Gower DJ. 2016. Visual pigments, ocular filters and the evolution of snake vision. Mol Biol Evol. 33: 24832495.

Smith MA. 1943. The fauna of British India, Ceylon and Burma including the whole of the Indo-Chinese sub-region. Reptilia and Amphibia. Volume III. Serpentes. Taylor and Francis, London.

Srinivasulu C, Srinivasulu B, Molur S. 2014. The status and distribution of reptiles in the Western Ghats, India. Conservation Assessment and Management Plan 
(CAMP). Wildlife Information Laision Development Society, Coimbatore, Tamil Nadu.

Stamatakis A. 2006. RAxML-VI-HPC: maximum likelihood-based phylogenetic analyses with thousands of taxa and mixed models. Bioinformatics 22: 26882690.

Silvestro D, Michalak I. 2012. RAxML GUI: a graphical front-end for RAxML. Org Divers Evol. 12: 335-337.

Streicher JW, Wiens JJ. 2016. Phylogenomic analyses reveal novel relationships among snake families. Mol Phylogenet Evol. 100: 160-169.

Tamura K, Peterson D, Peterson N, Stecher G, Nei M, Kumar S. 2011. MEGA5: molecular evolutionary genetics analysis using maximum likelihood, evolutionary distance, and maximum parsimony methods. Mol Biol Evol. 28: 2731-2739.

Uetz P, Freed P, Hošek J (Eds.). 2018. The Reptile Database, http://www.reptiledatabase.org (accessed November 14, 2018).

Underwood G. 1967. A Contribution to the Classification of Snakes. British Museum (Natural History), London.

Vences M, Guayasamin JM, Miralles A, De La Riva I. 2013. To name or not to name: Criteria to promote economy of change in Linnaean classification schemes. Zootaxa 3636: 201-244. 
Vidal N. 2002. Colubroid systematics: evidence for an early appearance of the venom apparatus followed by extensive evolutionary tinkering. J. Toxicol. Toxin. Rev. 21: 21-41.

Vidal N, Delmas AS, David P, Cruaud C, Couloux A, Hedges SB. 2007. The phylogeny and classification of caenophidian snakes inferred from seven nuclear protein-coding genes. C R Biol. 330: 182-187.

Wallach V, Williams KL, Boundy J. 2014. Snakes of the World a Catalogue of living and extinct Species. CRC Press, Taylor \& Francis Group.

Whitaker R, Captain A. 2004. Snakes of India: The Field Guide. Draco Books, Chennai.

Wiens JJ, Kuczynski CA, Smith SA, Mulcahy DG, Sites JW, Townsend TM, Tod W. 2008. Branch Lengths, Support, and Congruence: Testing the Phylogenomic Approach with 20 Nuclear Loci in Snakes. Sys Biol. 57: 420-431.

Wilkinson M, San Mauro D, Sherratt E, Gower DJ. 2011. A nine-family classification of caecilians (Amphibia: Gymnophiona). Zootaxa 2874: 41-64.

Zaher H. 1999. Hemipenial morphology of the South American xenodontine snakes, with a proposal for a monophyletic Xenodontinae and a reappraisal of colubroid hemipenes. Bull Am Mus Nat Hist. 240: 1-168.

Zaher H, Grazziotin FG, Cadle JE, Murphy RW, Moura-Leite JCD, Bonatto SL. 2009. Molecular phylogeny of advanced snakes (Serpentes, Caenophidia) with an emphasis on South American Xenodontines: a revised classification and descriptions of new taxa. Pap Avulsos Zool. (São Paulo). 49: 115-153. 
Zaher H, Grazziotin FG, Graboski R, Fuentes RG, Sánchez-Martinez P, Montingelli GG, Zhang Y-P, Murphy RW. 2012. Phylogenetic relationships of the genus Sibynophis (Serpentes: Colubroidea). Pap Avulsos Zool. (São Paulo). 52: $141-149$. 


\section{FIGURES}

Figure 1. Pruned ML tree showing bootstrap support for the relationships of species in the family Pareidae. See Appendix 4 for the complete ML phylogeny including 507 taxa (493 species of snakes and 7 non-snake squamates).

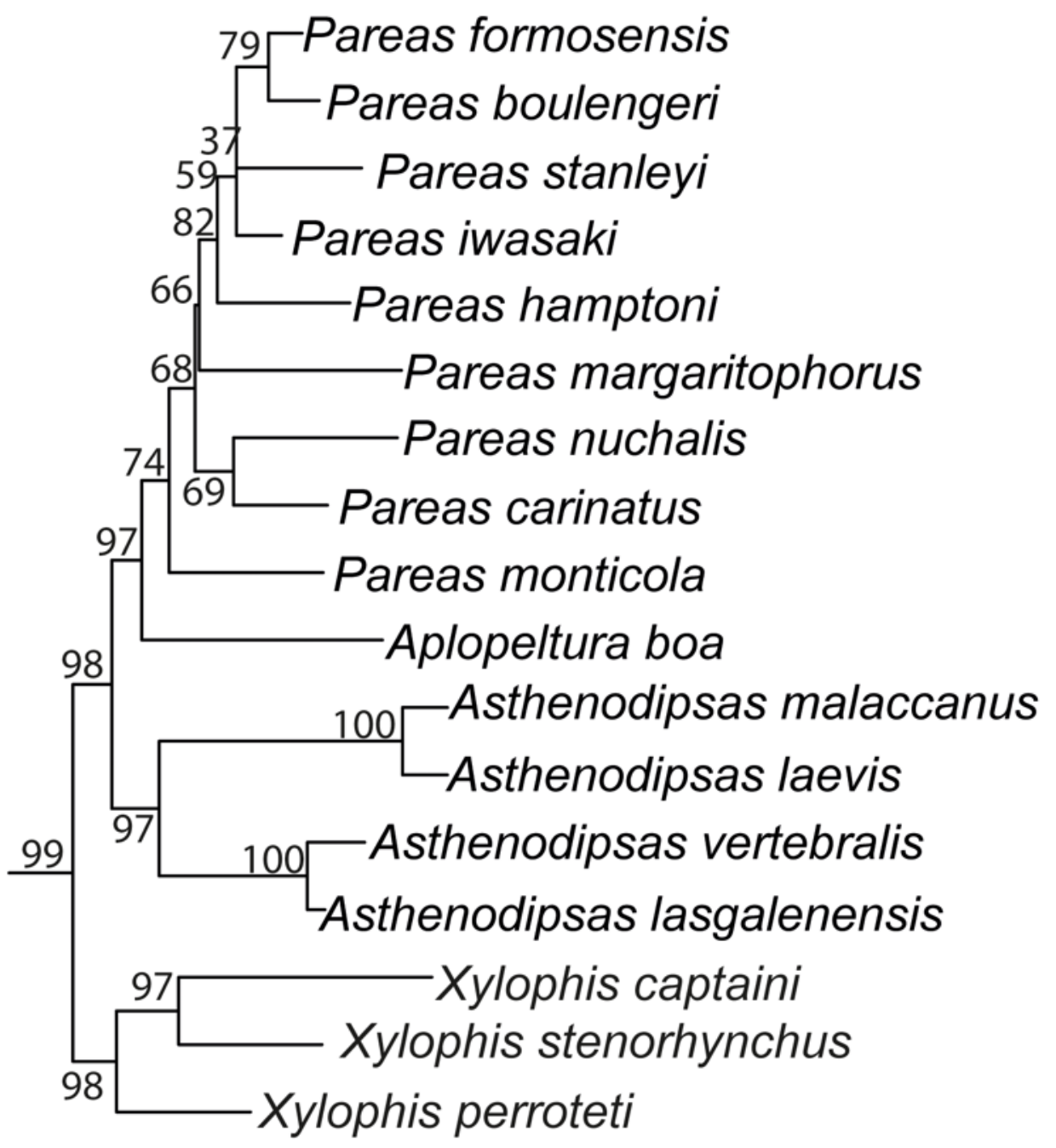

0.9 
Figure 2. Ranges of uncorrected p-distances (black \& grey) and K2P distances (dark blue \& light blue) for between-family (dark bars) and within-family (light bars) comparisons of snakes in the superfamily Colubroidea. Pareidae here includes Pareinae and Xylophiinae subfam. nov. Numbers on the $\mathrm{X}$ axis denotes sample size of subfamilies under each family.
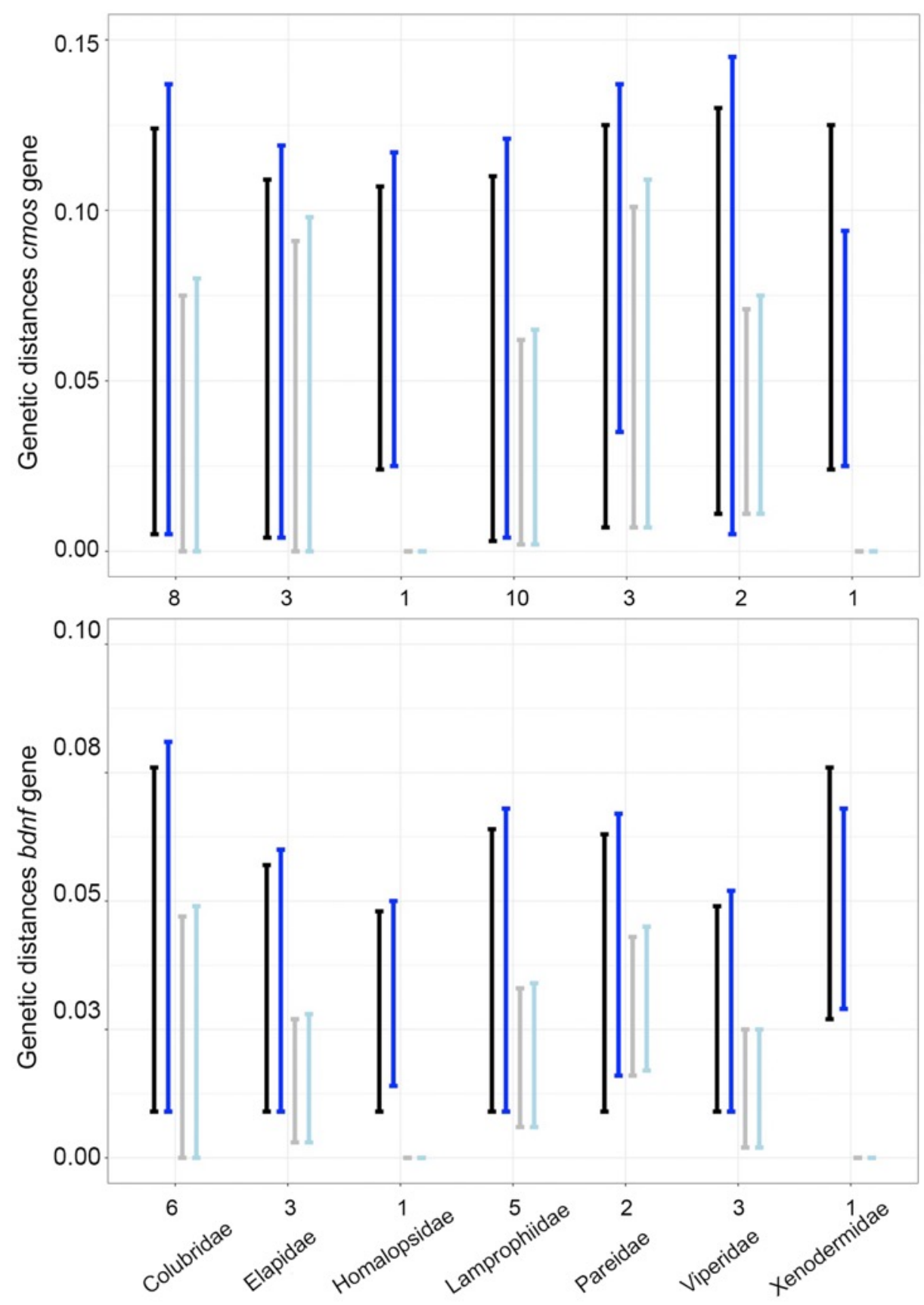
Figure 3. BEAST chronogram generated using concatenated-gene for all families and subfamilies of snakes. Numbers at internal branches indicate posterior probabilities. Error bars indicate 95\% Highest Posterior Densities for node ages. Nodes C1-C5 are the five calibrated nodes

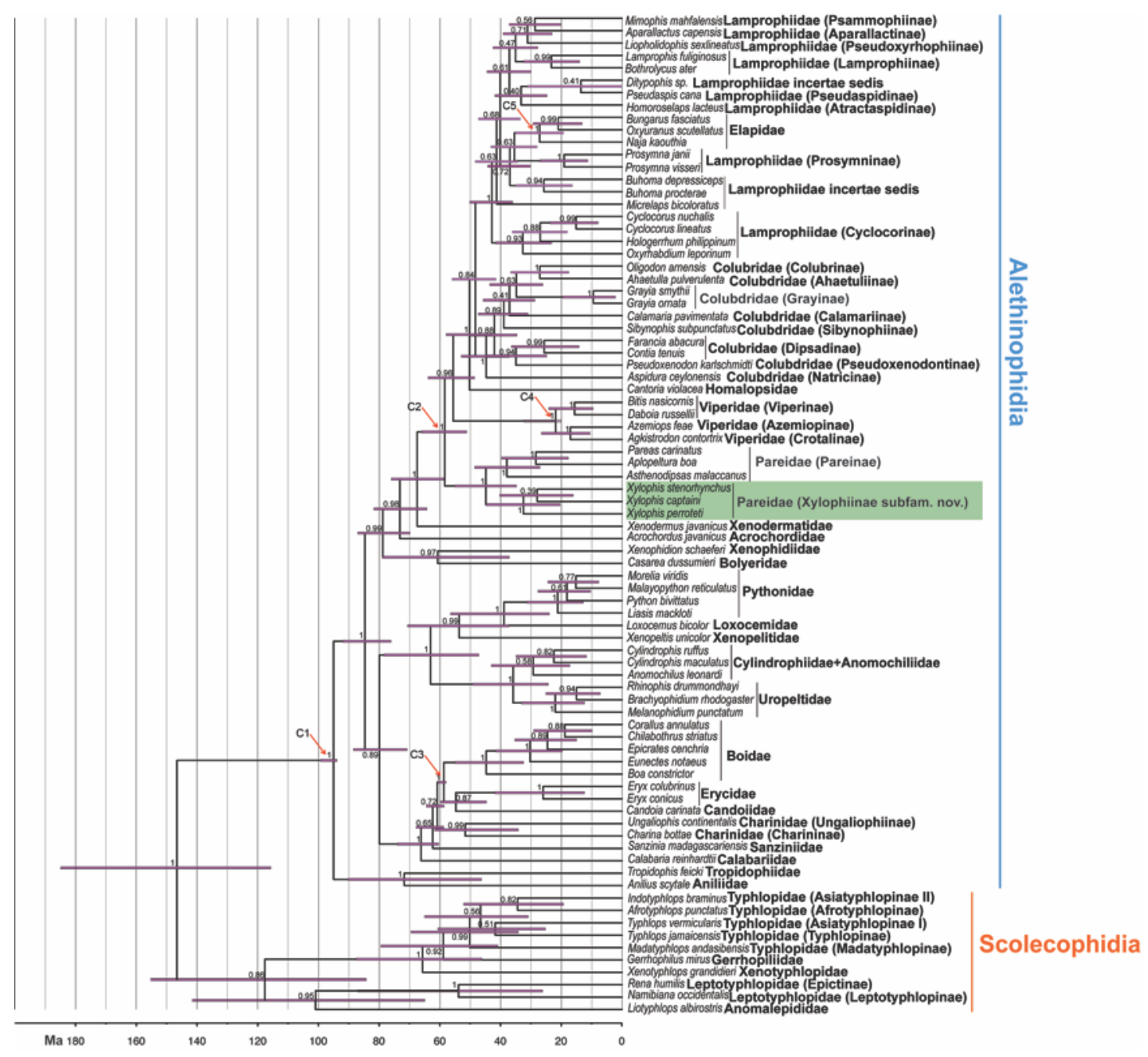


Figure 4. A. Geographic distribution of Xylophiinae subfam. nov. (green) and approximate distribution of subfamily Pareinae (blue). Photographs show representative taxa of the two subfamilies within Pareidae: B. Xylophis perroteti from Nilgiris, Tamil Nadu, India. C. Pareas monticola from Barail, Assam, India. Approximate distribution drawn based on locations provided in Srinivasulu et al (2014) and Wallach et al (2014).

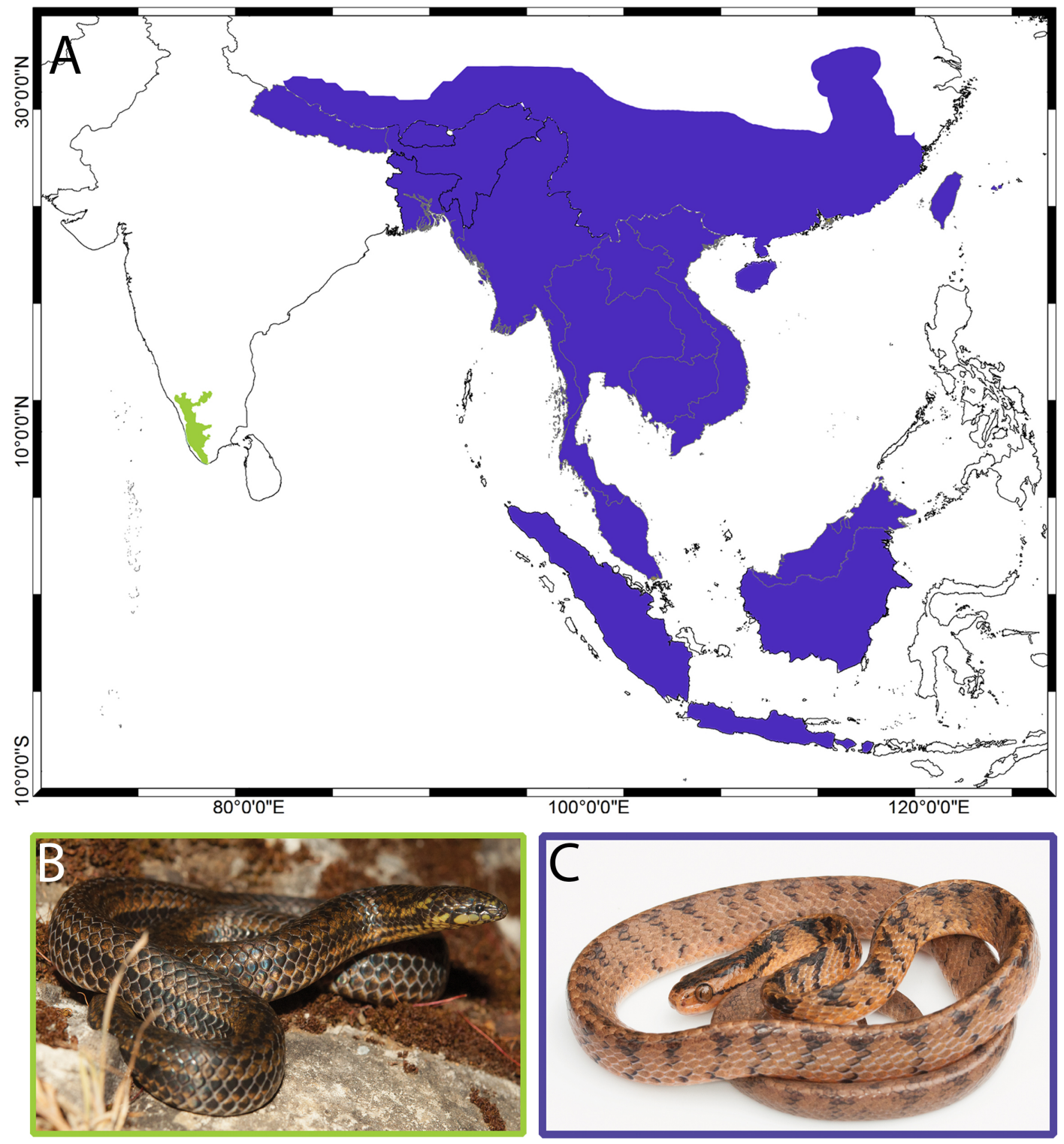


Table 1. GenBank accession and voucher numbers for gene sequences used in molecular dating analysis. * indicates data used in the "map to reference" analyses to identify homologous sequences from UCE data for the historical sample of $X$. stenorhynchus.

\begin{tabular}{|c|c|c|c|c|c|c|c|c|}
\hline sno & species & family (subfamily) & cytb & $16 \mathrm{~s}$ & $n d 4$ & cmos & bdnf & rag1 \\
\hline 1 & Acrochordus javanicus & Acrochordidae & _- & AF512745 & HM234055 & HM234058 & AY988036 & HM234061 \\
\hline 2 & Afrotyphlops punctatus & Typhlopidae (Afrotyphlopinae) & _- & _- & _- & _- & GU902395 & _ \\
\hline 3 & Agkistrodon contortrix & Viperidae (Crotalinae) & EU483383 & AF156566 & AF156577 & _ & EU402623 & EU402833 \\
\hline 4 & Ahaetulla pulverulenta & Colubridae (Ahaetuliinae) & KC347454 & KC347339 & KC347512 & KC347378 & _ & KC347416 \\
\hline 5 & Anilius scytale & Aniliidae & U69738 & FJ755180 & FJ755180 & AF544722 & EU402625 & AY988072 \\
\hline 6 & Anomochilus leonardi & Cylindrophiidae+Anomochiliidae & _ & AY953431 & _ & - & - & - \\
\hline 7 & Aparallactus capensis & Lamprophiidae (Aparallactinae) & AY188006 & AY188045 & FJ404331 & AY187967 & _- & _ \\
\hline 8 & Aplopeltura boa & Pareidae (Pareinae) & JF827673 & AF544787 & JF827650 & JF827696 & FJ433984 & _ \\
\hline 9 & Aspidura ceylonensis & Colubridae (Natricinae) & KC347477 & KC347361 & KC347527 & KC347400 & _- & KC347438 \\
\hline 10 & Asthenodipsas malaccanus & Pareidae (Pareinae) & KX660469 & KX660197 & KX660597 & KX660336 & _- & - \\
\hline 11 & Azemiops feae & Viperidae (Azemiopinae) & AY352747 & AF057234 & AY352808 & AF544695 & EU402628 & EU402836 \\
\hline 12 & Bitis nasicornis & Viperidae (Viperinae) & DQ305457 & AY188048 & DQ305475 & AY187970 & _ & KC330012 \\
\hline 13 & Boa constrictor & Boidae & AB177354 & AB177354 & AB177354 & AF544676 & KC330044 & KC347423 \\
\hline 14 & Boaedon fuliginosus & Lamprophiidae (Lamprophiinae) & AF471060 & AY188079 & FJ404365 & FJ404270 & EU402646 & EU402849 \\
\hline 15 & Bothrolycus ater & Lamprophiidae (Lamprophiinae) & AY612041 & AY611859 & AY611950 & FJ404347 & _ & _ \\
\hline 16 & Brachyophidium rhodogaster & Uropeltidae & _ & AY701023 & _ & - & _- & _ \\
\hline 17 & Buhoma depressiceps & Lamprophiidae incertae sedis & AY612042 & AY611860 & _ & AY611951 & _- & _- \\
\hline
\end{tabular}




\begin{tabular}{|c|c|c|c|c|c|c|c|c|}
\hline 18 & Buhoma procterae & Lamprophiidae incertae sedis & AY612001 & AY611818 & DQ486328 & AY611910 & - & - \\
\hline 19 & Bungarus fasciatus & Elapidae & EU579523 & EU579523 & EU579523 & AY058924 & FJ433989 & - \\
\hline 20 & Calabaria reinhardtii & Calabariidae & AY099985 & Z46494 & _ & AF544682 & EU402631 & EU402839 \\
\hline 21 & Calamaria pavimentata & Colubridae (Calamariinae) & AF471081 & KX694624 & - & AF471103 & FJ434005 & - \\
\hline 22 & Candoia carinata & Candoiidae & AY099984 & EU419850 & - & AY099961 & FJ433974 & AY988065 \\
\hline 23 & Cantoria violacea & Homalopsidae & EF395897 & KX694627 & EF395922 & - & - & - \\
\hline 24 & Casarea dussumieri & Bolyeridae & U69755 & AF544827 & - & AF544731 & EU402632 & EU402840 \\
\hline 25 & Charina bottae & Charinidae (Charininae) & AY099986 & AF544816 & AF302959 & AY099971 & FJ433978 & AY988076 \\
\hline 26 & Chilabothrus striatus & Boidae & _- & - & KC329966 & KC329991 & KC330056 & KC330027 \\
\hline 27 & Contia tenuis & Colubridae (Dipsadinae) & GU112384 & AY577030 & GU112419 & AF471134 & GU112346 & - \\
\hline 28 & Corallus annulatus & Boidae & KC750012 & - & KC750018 & KC750007 & JX576167 & KC750047 \\
\hline 29 & Cyclocorus nuchalis & Lamprophiidae (Cyclocorinae) & MG458754 & - & - & MG458764 & - & - \\
\hline 30 & Cyclocorus lineatus & Lamprophiidae (Cyclocorinae) & MG458750 & - & - & MG458759 & - & - \\
\hline 31 & Cylindrophis maculatus & Cylindrophiidae+Anomochilidae & KC347460 & KC347355 & KC347494 & KC347395 & - & KC347433 \\
\hline 32 & Cylindrophis ruffus & Cylindrophiidae+Anomochilidae & AB179619 & AB179619 & AB179619 & AF471133 & AY988037 & AY988071 \\
\hline 33 & Daboia russellii & Viperidae (Viperinae) & EU913478 & EU913478 & EU913478 & AF471156 & EU402636 & EU402843 \\
\hline 34 & Ditypophis sp. & Lamprophiidae & - & - & - & - & JQ073079 & JQ073200 \\
\hline 35 & Epicrates cenchria & Boidae & HQ399501 & - & KC329975 & KC330008 & KC330073 & - \\
\hline 36 & Eryx colubrinus & Erycidae & U69811 & AF544819 & - & AF544716 & EU402639 & DQ465571 \\
\hline 37 & Eryx conicus & Erycidae & GQ225658 & AF512743 & GQ225672 & - & - & AY988074 \\
\hline 38 & Eunectes notaeus & Boidae & HQ399499 & AM236347 & KC329978 & HQ399536 & KC330076 & HQ399516 \\
\hline 39 & Farancia abacura & Colubridae (Dipsadinae) & U69832 & Z46491 & U49307 & AF471141 & _- & KR814740 \\
\hline
\end{tabular}




\begin{tabular}{|c|c|c|c|c|c|c|c|c|}
\hline 40 & Gerrhopilus mirus & Gerrhopilidae & AM236345 & AM236345 & AM236345 & - & GU902394 & - \\
\hline 41 & Grayia ornata & Colubridae (Grayiinae) & - & AF158503 & AF544663 & AF544684 & FJ434002 & - \\
\hline 42 & Grayia smythii & Colubridae (Grayiinae) & DQ112077 & - & DQ112080 & - & - & - \\
\hline 43 & Hologerrhum philippinum & Lamprophiidae (Cyclocorinae) & MG458758 & - & - & MG458766 & - & - \\
\hline 44 & Homoroselaps lacteus & Lamprophiidae (Atractaspidinae) & AY611992 & AY611809 & FJ404338 & AY611901 & JQ599029 & - \\
\hline 45 & Indotyphlops braminus & Typhlopidae (Asiatyphlopinae II) & DQ343649 & - & - & AF544717 & FJ433959 & - \\
\hline 46 & Liasis mackloti & Pythonidae & U69839 & EF545051 & - & AF544726 & FJ433970 & - \\
\hline 47 & Liopholidophis sexlineatus & Lamprophiidae (Pseudoxyrhophiinae) & DQ979985 & AY188063 & FJ404373 & AY187985 & - & - \\
\hline 48 & Liotyphlops albirostris & Anomalepididae & AF544672 & AF366762 & - & AF544727 & EU402650 & EU402853 \\
\hline 49 & Loxocemus bicolor & Loxocemidae & AY099993 & AF544828 & - & AY444035 & EU402651 & - \\
\hline 50 & Madatyphlops andasibensis & Typhlopidae (Madatyphlopinae) & - & - & - & - & GU902453 & JQ073249 \\
\hline 51 & Malayopython reticulatus & Pythonidae & U69860 & EF545062 & - & AF544675 & FJ433969 & EU624119 \\
\hline 52 & Melanophidium punctatum & Uropeltidae & - & AY701024 & - & - & - & - \\
\hline 53 & Micrelaps bicoloratus & Lamprophiidae (Aparallactinae) & DQ486349 & - & - & DQ486173 & - & - \\
\hline 54 & Mimophis mahfalensis & Lamprophiidae (Psammophiinae) & DQ486461 & AY188070 & - & AY187992 & JQ073081 & - \\
\hline 55 & Morelia viridis & Pythonidae & EF545098 & EF545048 & - & - & - & - \\
\hline 56 & Naja kaouthia & Elapidae & FR693728 & GQ359757 & EU624209 & AY058938 & EU402654 & EU402857 \\
\hline 57 & Namibiana occidentalis & Leptotyphlopidae (Leptotyphlopinae) & - & GQ469251 & - & GQ469074 & GQ469189 & - \\
\hline 58 & Oligodon arnensis & Colubridae (Colubrinae) & KC347464 & KC347365 & KC347504 & KC347404 & - & KC347442 \\
\hline 59 & Oxyrhabdium leporinum & Lamprophiidae (Cyclocorinae) & AF471029 & - & - & DQ112081 & - & - \\
\hline 60 & Oxyuranus scutellatus & Elapidae & EU547051 & EU547149 & EF210827 & EU546916 & - & - \\
\hline 61 & Pareas carinatus & Pareidae (Pareinae) & JF827677 & AF544802 & JF827653 & JF827702 & FJ433985 & - \\
\hline
\end{tabular}




\begin{tabular}{|c|c|c|c|c|c|c|c|c|}
\hline 62 & Prosymna janii & Lamprophiidae (Prosymninae) & FJ404319 & FJ404222 & FJ404389 & FJ404293 & _ & - \\
\hline 63 & Prosymna visseri & Lamprophiidae (Prosymninae) & AY188033 & AY188072 & _ & AY187994 & - & - \\
\hline 64 & Pseudaspis cana & Lamprophiidae (Pseudaspidinae) & AY612080 & AY611898 & DQ486319 & DQ486167 & - & - \\
\hline 65 & Pseudoxenodon karlschmidti & Colubridae (Pseudoxenodontinae) & AF471080 & JF697330 & _ & AF471102 & JQ599045 & - \\
\hline 66 & Python bivittatus & Pythonidae & JX401131 & KF010492 & - & AF435016 & XM7433022 & - \\
\hline 67 & Rena humilis & Leptotyphlopidae (Epictinae) & AY099991 & AB079597 & AB079597 & AY099979 & - & - \\
\hline 68 & Rhinophis drummondhayi & Uropeltidae & AF544673 & AY701028 & - & AF544719 & FJ433966 & - \\
\hline 69 & Sanzinia madagascariensis & Sanziniidae & U69866 & AY336066 & - & EU403580 & AY988033 & AY988067 \\
\hline 70 & Sibynophis subpunctatus & Colubridae (Sibynophiinae) & KC347471 & KC347373 & KC347516 & KC347411 & - & KC347449 \\
\hline 71 & Tropidophis feicki & Tropidophiidae & KF811124 & AF512733 & - & KF811110 & KF811074 & - \\
\hline 72 & Typhlops jamaicensis & Typhlopidae (Typhlopinae) & KF993259 & AF366764 & - & AF544733 & EU402664 & EU402866 \\
\hline 73 & Ungaliophis continentalis & Charinidae (Ungaliophiinae) & U69870 & AF544833 & - & AF544724 & EU402665 & EU402867 \\
\hline 74 & Xenodermus javanicus & Xenodermidae & - & AF544810 & U49320 & AF544711 & EU402667 & EU402869 \\
\hline 75 & Xenopeltis unicolor & Xenopeltidae & AB179620 & AB179620 & AB179620 & AF544689 & EU402668 & DQ465564 \\
\hline 76 & Xenophidion schaeferi & Xenophidiidae & AY574279 & - & - & - & - & - \\
\hline 77 & Xenotyphlops grandidieri & Xenotyphlopidae & KF770844 & - & - & - & GU902457 & - \\
\hline 78 & Xerotyphlops vermicularis & Typhlopidae (Asiatyphlopinae I) & JQ910544 & - & - & - & GU902397 & - \\
\hline 79 & Xylophis perroteti & Pareidae (Xylophiinae subfam. nov.) & - & MK340908* & MK340910* & MK344193* & MK344197* & MK340913* \\
\hline 80 & Xylophis stenorhynchus & Pareidae (Xylophiinae subfam. nov.) & MK340915 & MK340907 & MK340911 & MK344194 & MK344198 & - \\
\hline 81 & Xylophis captaini & Pareidae (Xylophiinae subfam. nov.) & MK340914* & MK340909* & MK340912 & MK344195 & MK344196 & - \\
\hline
\end{tabular}

*indicates data used in the 'map to reference' analyses to identify homologous sequences from UCE data for the historical sample of $X$. stenorhynchus. 


\section{Supplementary material}

Figure S1. RAxML trees for concatenated data for 81 taxa, with three different alignment treatments for $16 \mathrm{~s}$.

A) ML tree for concatenated data including $16 \mathrm{~s}$ aligned by ClustalW, with all sites included. Numbers on tree are bootstrap values (based on 1,000 replicates). Scale bar indicates substitutions per site.

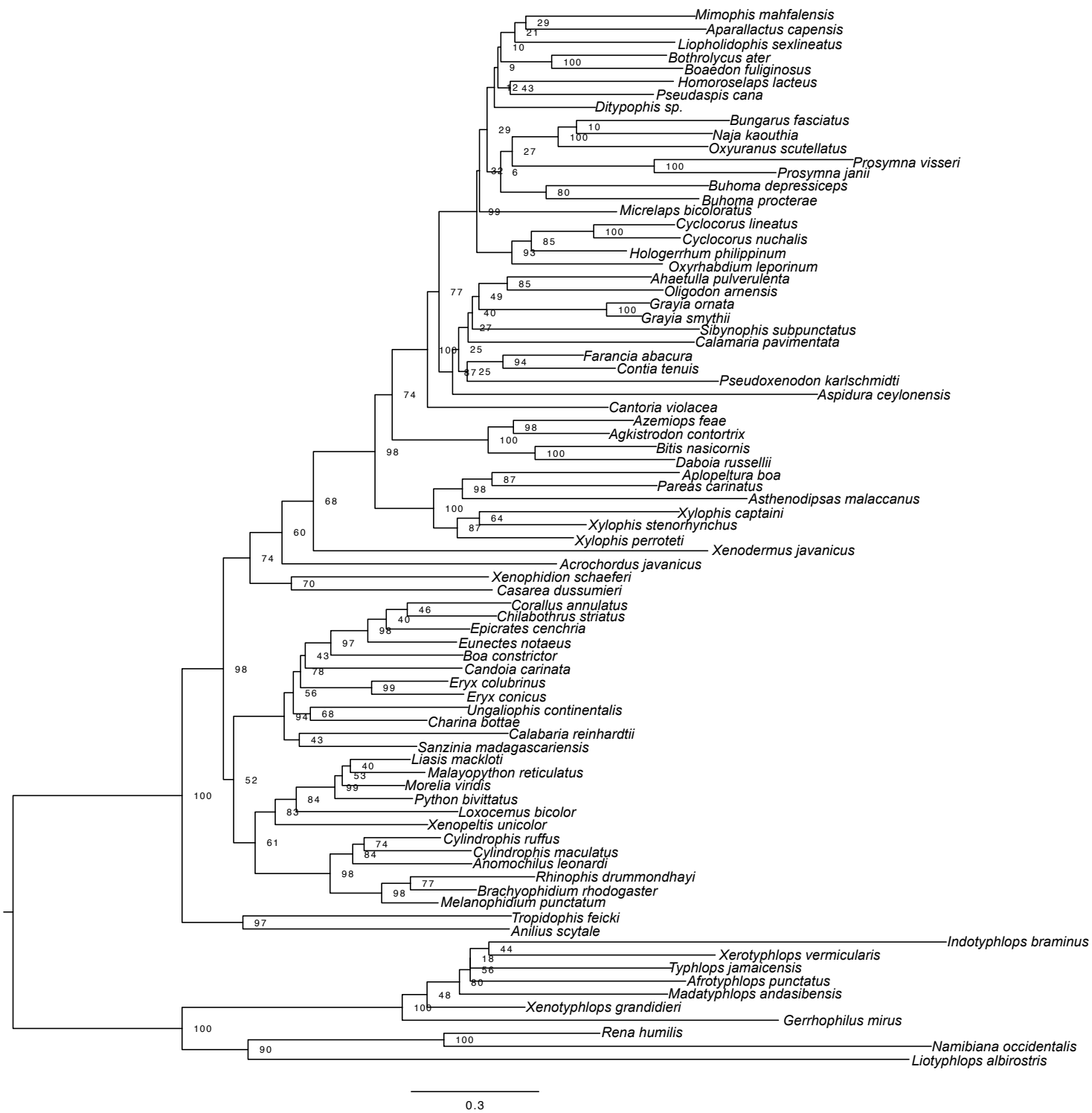


B) ML tree for concatenated data including $16 \mathrm{~s}$ aligned by MUSCLE, with all sites included. Numbers on tree are bootstrap values (based on 1,000 replicates). Scale bar indicates substitutions per site.

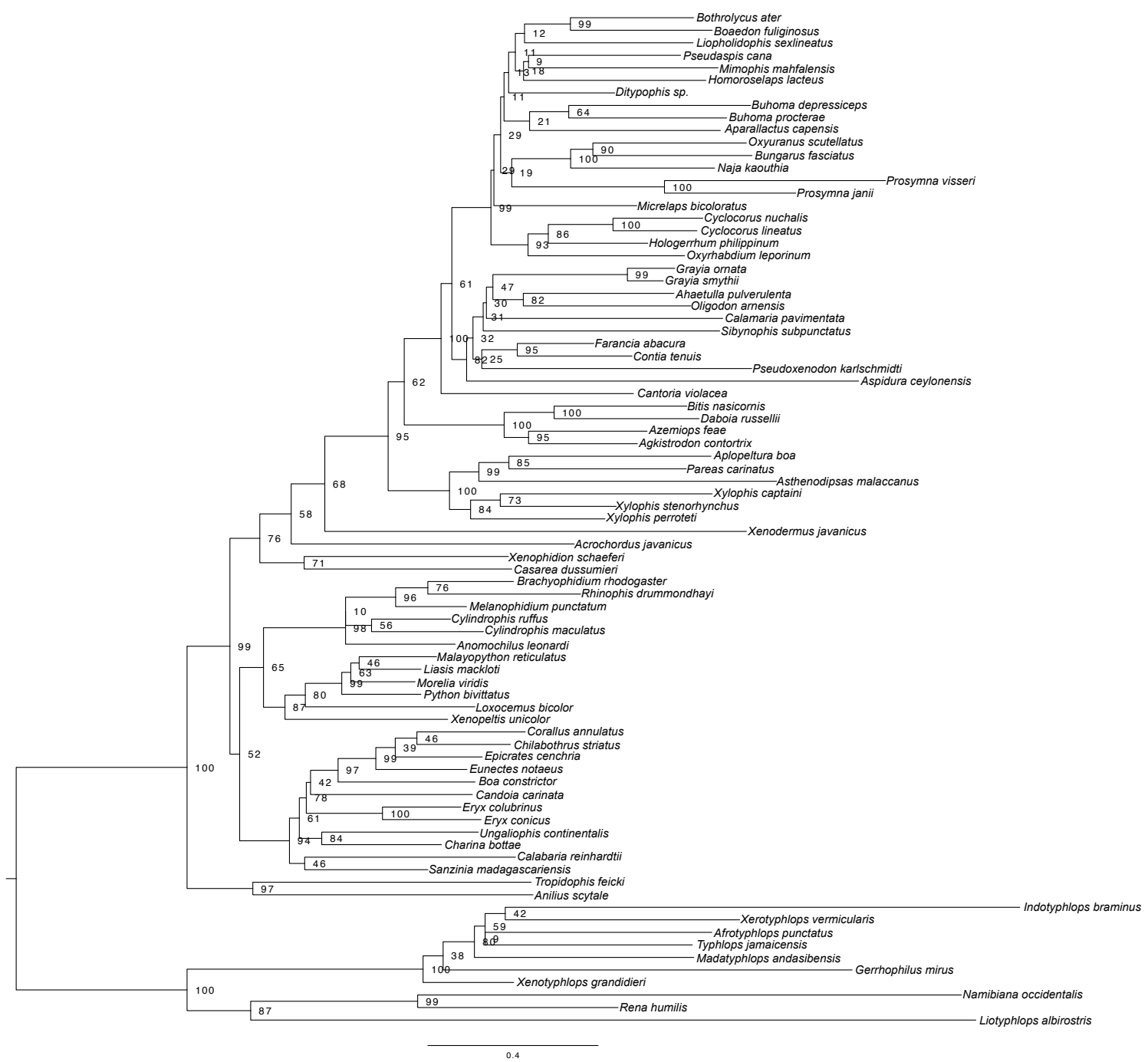


C) ML tree for concatenated data including $16 \mathrm{~s}$ aligned by ClustalW, with ambiguously aligned sites removed under 'less stringent' option in Gblocks. Numbers on tree are bootstrap values (based on 1,000 replicates). Scale bar indicates substitutions per site.

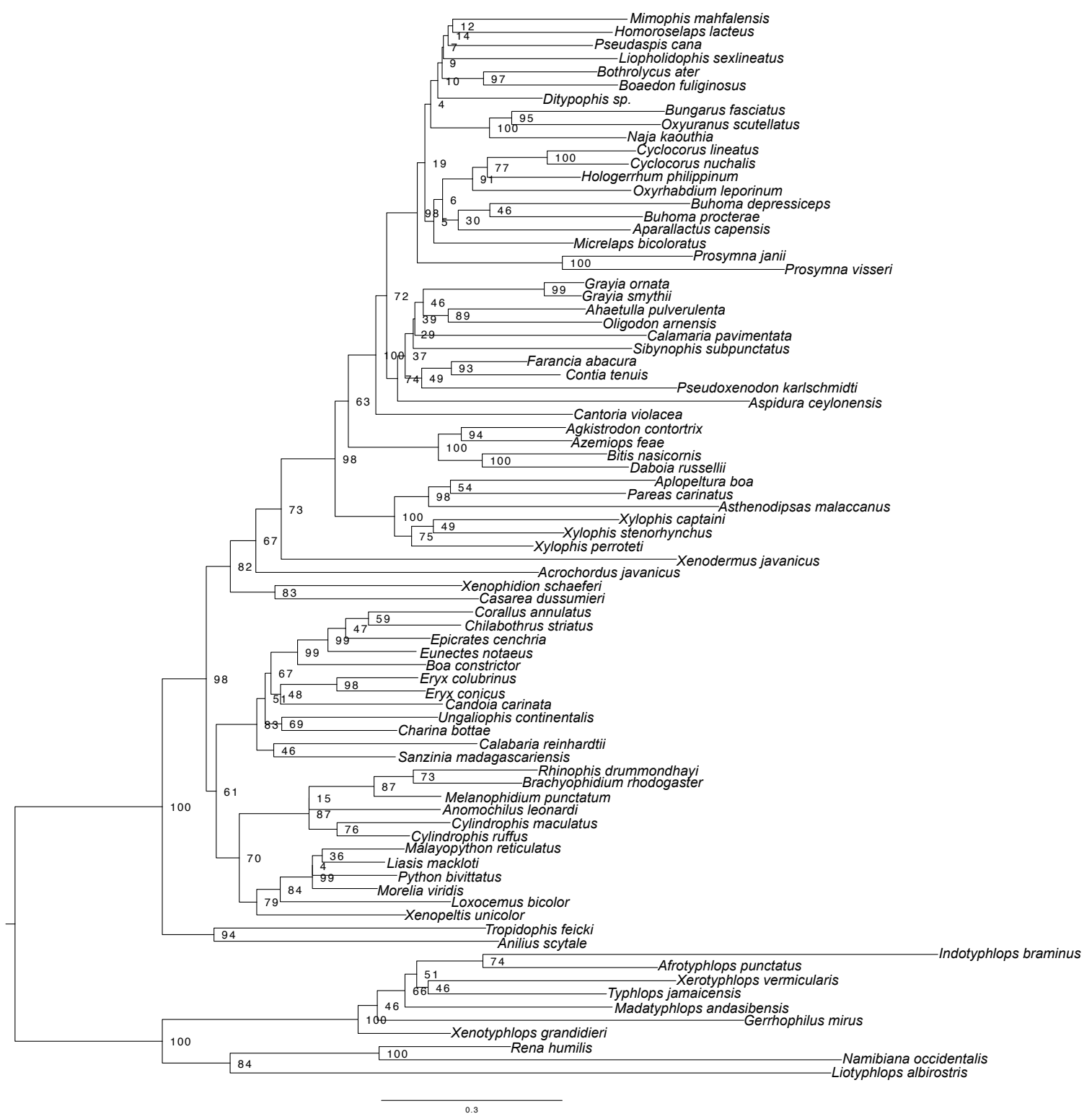


Figure S2. RAxML phylogeny highlighting relationships of the genus Xylophis.

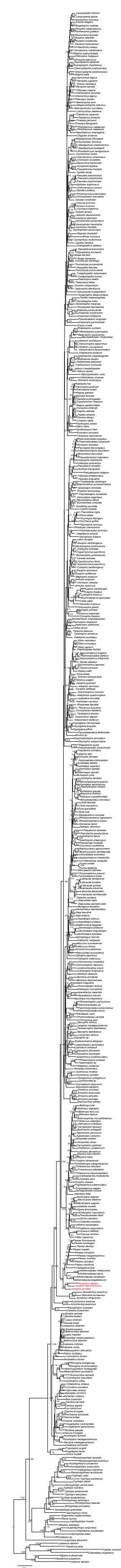


Figure S3. BEAST chronograms generated using concatenated gene sequence data for all families and subfamilies of snakes (81 taxa).

A. Concatenated dataset including all sites. Numbers at internal nodes indicate mean estimated node ages (and 95\% highest posterior densities)

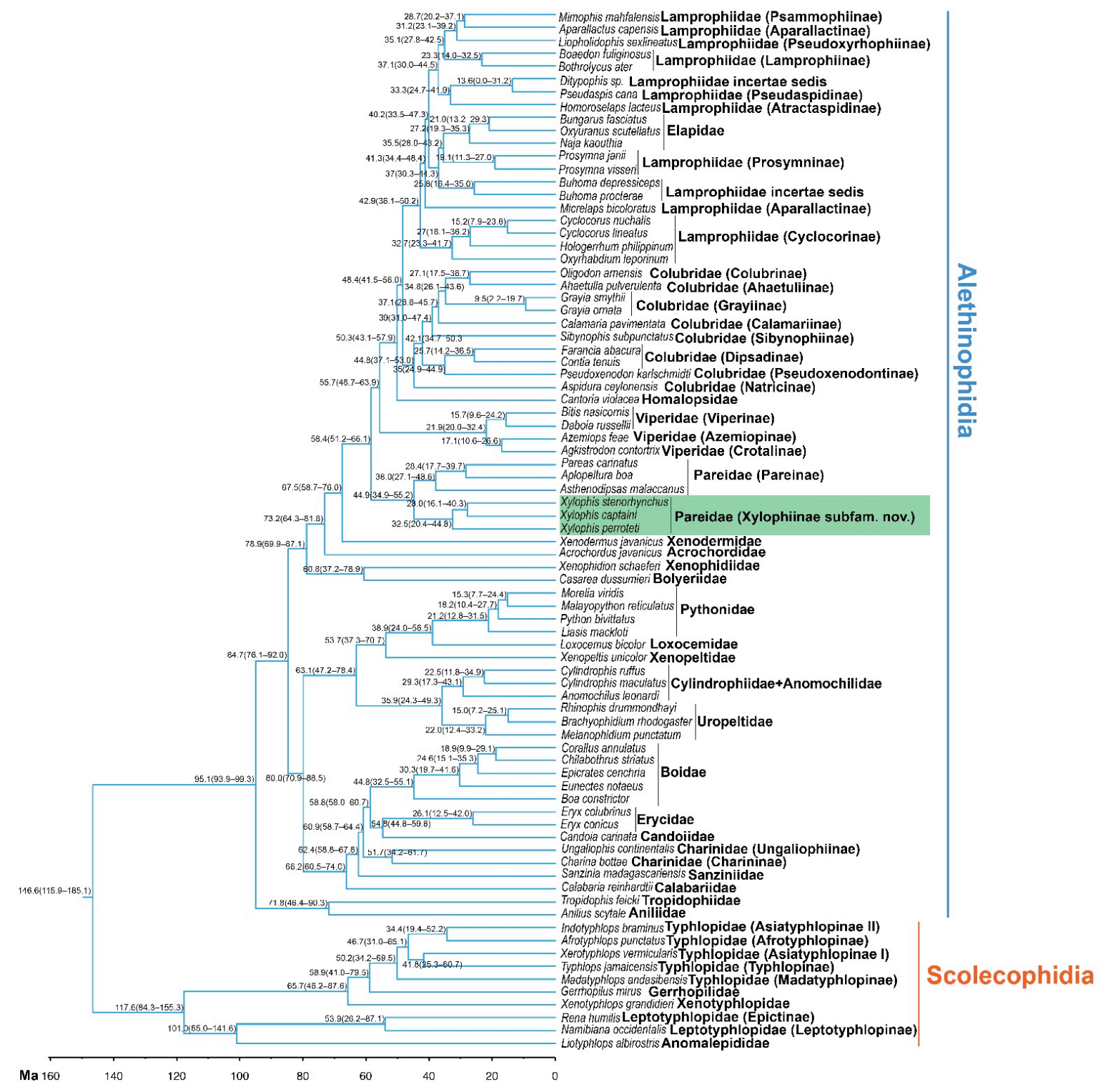


B.Concatenated dataset in which third codon position of the mitochondrial genes (nd4 and cytb) are excluded. Numbers at internal nodes indicate mean estimated node ages (and 95\% highest posterior densities)

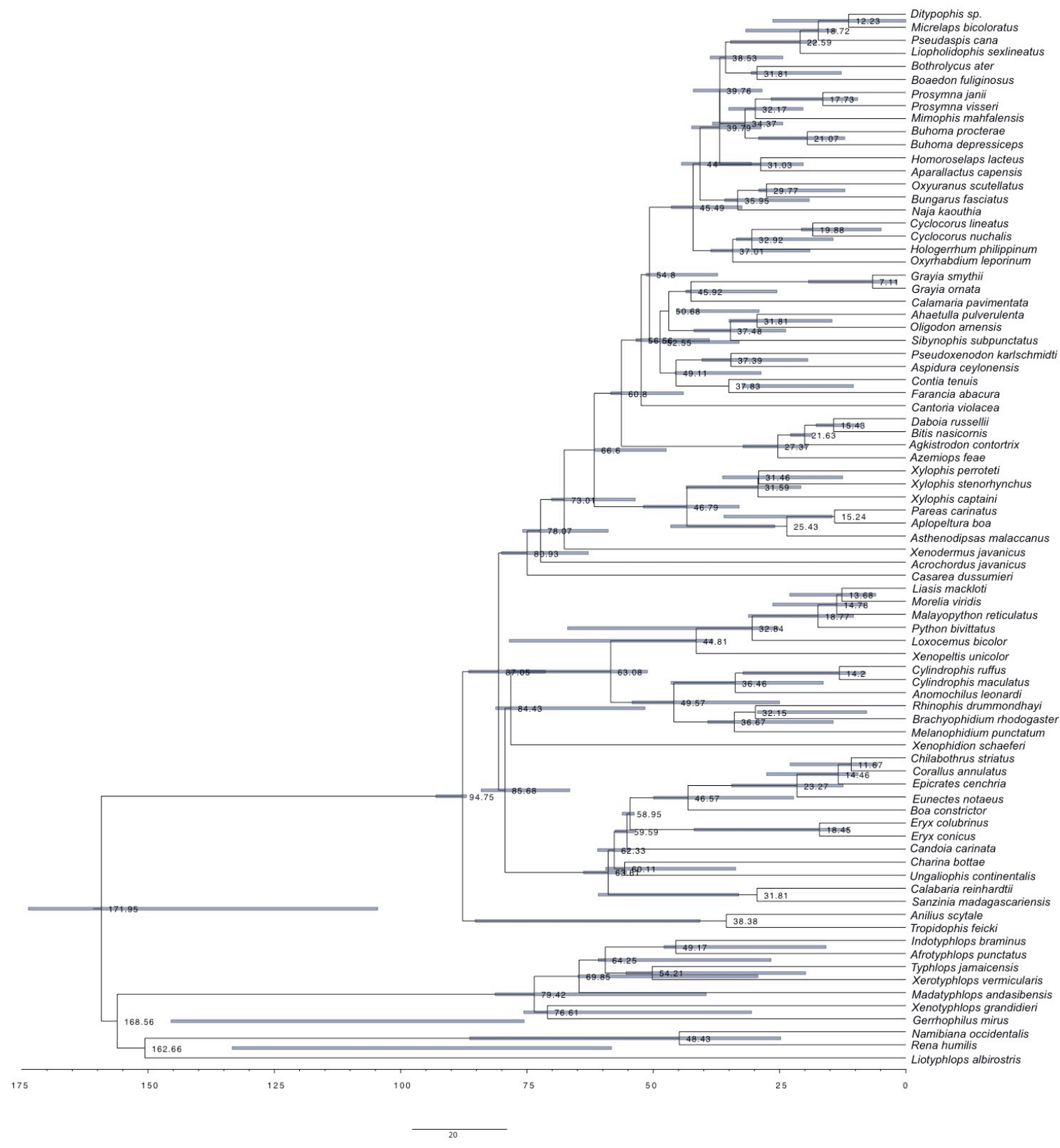


Figure S4. Comparison of estimated mean divergence dates from BEAST analyses of data in which third codon positions of mitochondrial genes ( $n d 4$ and $c y t b$ ) are either included or excluded. Nodes C1-C5 are the five calibrated nodes; Node 42 is the divergence between Pareinae and Xylophiinae subfam. nov. Points are plotted for the 57 out of 80 nodes in common between the two BEAST trees (see Fig. S3).

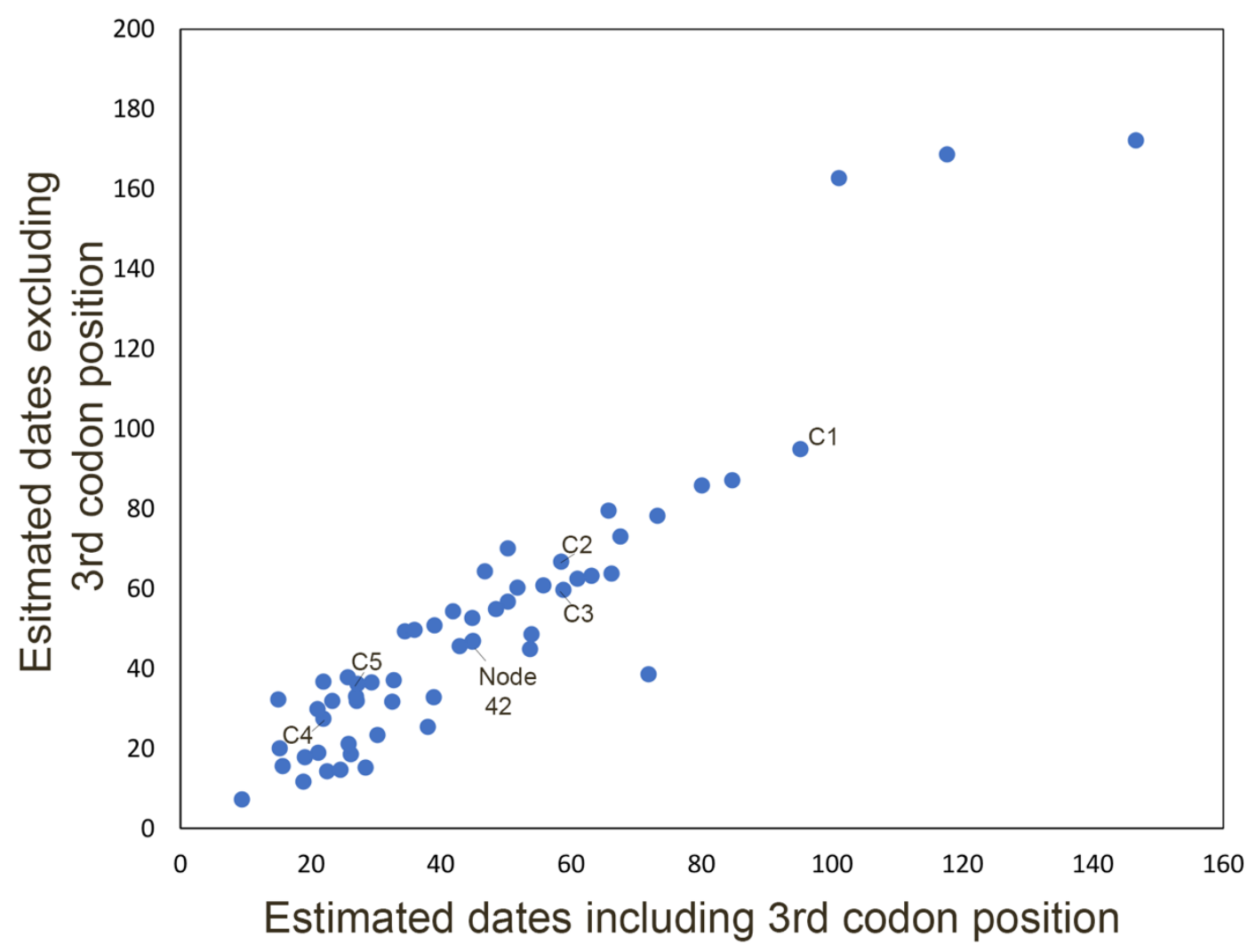


Table S1. GenBank accession and voucher numbers for gene sequences used in Maximum Likelihood analysis.

\begin{tabular}{|c|c|c|c|c|c|c|c|}
\hline sno & Species & CYTB & $16 \mathrm{~s}$ & ND4 & cMOs & BDNF & RAG1 \\
\hline 1 & Acanthophis laevis & AY340138 & - & AY340167 & - & - & - \\
\hline 2 & Acanthophis praelongus & EU547063 & EU547161 & AY340164 & EU546926 & - & - \\
\hline 3 & Achalinus meiguensis & FJ424614 & FJ424614 & FJ424614 & - & - & - \\
\hline 4 & Acrantophis dumerili & EU403574 & EU419794 & - & EU403581 & AY988032 & AY988066 \\
\hline 5 & Acrantophis madagascariensis & U69736 & AY336071 & - & EU403578 & FJ433973 & - \\
\hline 6 & Acrochordus arafurae & - & - & HM234056 & - & - & HM234062 \\
\hline 7 & Acrochordus javanicus & - & AF512745 & HM234055 & HM234058 & AY988036 & HM234061 \\
\hline 8 & Acutotyphlops kunuaensis & - & - & & - & GU902419 & - \\
\hline 9 & Acutotyphlops subocularis & JQ910524 & KF993127 & - & - & GU902418 & - \\
\hline 10 & Adelophis foxi & KF258652 & - & KF258635 & - & KF258601 & - \\
\hline 11 & Adelphicos quadrivirgatus & GQ895853 & - & - & GQ895796 & - & - \\
\hline 12 & Afronatrix anoscopus & - & - & AF420076 & AF 471123 & EU402622 & EU402832 \\
\hline 13 & Afrotyphlops punctatus & - & - & & - & GU902395 & - \\
\hline 14 & Agkistrodon contortrix & EU483383 & AF 156566 & AF156577 & - & EU402623 & EU402833 \\
\hline 15 & Agkistrodon piscivorus & EU483436 & AF057278 & AF 156578 & AF471096 & JQ599004 & - \\
\hline 16 & Ahaetulla mycterizans & KX660479 & KX660205 & KX660604 & KX660345 & - & - \\
\hline 17 & Ahaetulla pulverulenta & KC347454 & KC347339 & KC347512 & KC347378 & - & KC347416 \\
\hline 18 & Aipysurus apraefrontalis & KC014380 & JX423420 & JX002981 & KC014288 & - & _- \\
\hline 19 & Aipysurus duboisii & JX423393 & JX423423 & JX423411 & FJ587163 & - & - \\
\hline 20 & Alsophis anomalus & - & FJ666092 & - & - & - & - \\
\hline 21 & Alsophis antillensis & FJ416726 & FJ416702 & FJ416800 & - & JQ599005 & - \\
\hline 22 & Alsophis cantherigerus & AF544669 & AF 158475 & - & - & - & - \\
\hline 23 & Alsophis portoricensis & - & AF 158517 & - & - & - & - \\
\hline 24 & Amastridium sapperi & GQ334479 & - & GQ334580 & - & - & - \\
\hline 25 & Amastridium veliferum & - & - & - & GQ895797 & - & - \\
\hline 26 & Amphiesma craspedogaster & JQ687429 & - & JQ687412 & - & - & - \\
\hline 27 & Amphiesma sauteri & AF402905 & - & - & - & - & - \\
\hline 28 & Amphiesma stolatum & JQ687432 & - & JQ687425 & KJ685661 & EU402624 & - \\
\hline 29 & Amplorhinus multimaculatus & DQ486340 & AY611880 & DQ486316 & DQ486164 & - & - \\
\hline 30 & Anilius scytale & U69738 & FJ755180 & FJ755180 & AF544722 & EU402625 & AY988072 \\
\hline 31 & Anomochilus leonardi & - & AY953431 & - & - & - & - \\
\hline 32 & Antaresia childreni & - & EF545058 & - & AY099967 & - & - \\
\hline 33 & Antillophis andreae & - & AF 158511 & - & - & - & - \\
\hline 34 & Antillophis parvifrons & - & AF 158510 & - & - & JQ599006 & - \\
\hline 35 & Aparallactus capensis & AY188006 & AY188045 & FJ404331 & AY187967 & - & - \\
\hline 36 & Aparallactus werneri & AF471035 & - & U49315 & AF471116 & EU402626 & - \\
\hline 37 & Aplopeltura boa & JF827673 & AF544787 & JF827650 & JF827696 & FJ433984 & - \\
\hline 38 & Apostolepis dimidiata & JQ598917 & GQ457725 & - & GQ457844 & JQ599008 & - \\
\hline 39 & Arizona elegans & DQ902101 & - & DQ902279 & DQ902058 & - & - \\
\hline 40 & Arrhyton dolichura & FJ416721 & AF 158507 & FJ416795 & - & - & - \\
\hline 41 & Arrhyton exiguum & - & FJ416705 & - & - & - & - \\
\hline 42 & Aspidelaps lubricus & FR693724 & - & - & - & - & - \\
\hline 43 & Aspidelaps scutatus & - & AY188046 & AY058969 & AY187968 & - & - \\
\hline 44 & Aspidites melanocephalus & U69741 & EF545060 & - & DQ465557 & EU402627 & DQ465560 \\
\hline 45 & Aspidomorphus lineaticollis & - & GQ397237 & - & GQ397227 & - & - \\
\hline
\end{tabular}




\begin{tabular}{|c|c|c|c|c|c|c|c|}
\hline 46 & Aspidura ceylonensis & KC347477 & KC347361 & KC347527 & KC347400 & - & KC347438 \\
\hline 47 & Asthenodipsas laevis & KX660468 & KX660196 & KX660596 & KX660335 & _- & _ \\
\hline 48 & Asthenodipsas lasgalenensis & KC916763 & - & - & - & - & - \\
\hline 49 & Asthenodipsas malaccanus & KX660469 & KX660197 & KX660597 & KX660336 & - & - \\
\hline 50 & Asthenodipsas vertebralis & KC916750 & - & - & - & - & - \\
\hline 51 & Atheris squamigera & AJ275684 & AF544788 & EU624212 & AF544734 & _- & _ \\
\hline 52 & Atractaspis irregularis & - & - & & - & JN614212 & - \\
\hline 53 & Atractaspis micropholis & AY612006 & AY611823 & FJ404336 & AY611915 & FJ433994 & _ \\
\hline 54 & Atractus albuquerquei & JQ598918 & GQ457726 & _ & GQ457845 & JQ599009 & _ \\
\hline 55 & Atractus wagleri & GQ334480 & - & GQ334581 & - & - & - \\
\hline 56 & Atretium schistosum & KC347487 & - & KC347525 & KC347383 & - & KC347421 \\
\hline 57 & Atretium yunnanensis & GQ281787 & - & JQ687423 & JQ687448 & - & - \\
\hline 58 & Atropoides olmec & AY223585 & AY223669 & AY223632 & - & - & - \\
\hline 59 & Atropoides picadoi & AY220324 & AF057255 & AY220347 & - & - & - \\
\hline 60 & Austrelaps labialis & EU547077 & EU547175 & EU547029 & EU546939 & - & - \\
\hline 61 & Austrelaps superbus & EU547078 & EU547176 & EU547030 & EU546940 & - & - \\
\hline 62 & Azemiops feae & AY352747 & AF057234 & AY352808 & AF544695 & EU402628 & EU402836 \\
\hline 63 & Balanophis ceylonensis & KC347474 & KC347344 & KC347520 & KC347384 & _- & KC347422 \\
\hline 64 & Bitis nasicornis & DQ305457 & AY188048 & DQ305475 & AY187970 & - & KC330012 \\
\hline 65 & Boa constrictor & AB177354 & AB177354 & AB177354 & AF544676 & KC330044 & KC347423 \\
\hline 66 & Bogertophis rosaliae & DQ902102 & - & DQ902280 & DQ902059 & - & - \\
\hline 67 & Boiga barnesii & KC347469 & KC347345 & KC347499 & KC347385 & - & - \\
\hline 68 & Boiga siamensis & KX660527 & KX660257 & KX660645 & KX660398 & - & - \\
\hline 69 & Borikenophis portoricensis & AF471085 & - & FJ416806 & AF471126 & JQ599012 & - \\
\hline 70 & Bothriechis schlegeliil & KC847272 & KC847257 & KC847285 & - & FJ433983 & - \\
\hline 71 & Bothrochilus albertisii & - & - & - & KF811096 & KF811059 & KF811160 \\
\hline 72 & Bothrocophias microphthalmus & FR691567 & AY223670 & FR691540 & - & - & - \\
\hline 73 & Bothrops leucurus & EU867279 & EU867267 & AF246283 & - & - & - \\
\hline 74 & Bothrops lojanus & FR691566 & - & FR691536 & - & - & - \\
\hline 75 & Bothrops lutzi & KF801130 & - & KF801261 & - & - & - \\
\hline 76 & Bothrops oligolepis & - & KX660260 & KX660646 & - & - & - \\
\hline 77 & Brachyophidium rhodogaster & - & AY701023 & - & - & - & - \\
\hline 78 & Brachyorrhos raffrayi & JX139713 & - & - & - & - & - \\
\hline 79 & Brachyurophis approximans & - & KF736330 & - & - & - & - \\
\hline 80 & Brachyurophis semifasciata & EU547057 & EU547155 & EU547011 & EU546921 & - & - \\
\hline 81 & Buhoma depressiceps & AY612042 & AY611860 & _ & AY611951 & - & - \\
\hline 82 & Buhoma procterae & AY612001 & AY611818 & DQ486328 & AY611910 & - & - \\
\hline 83 & Bungarus fasciatus & EU579523 & EU579523 & EU579523 & AY058924 & FJ433989 & - \\
\hline 84 & Caaeteboia amarali & JQ598921 & - & - & - & - & - \\
\hline 85 & Calabaria reinhardtii & AY099985 & Z46494 & - & AF544682 & EU402631 & EU402839 \\
\hline 86 & Calamaria pavimentata & AF471081 & KX694624 & - & AF471103 & FJ434005 & - \\
\hline 87 & Calamodontophis paucidens & - & GQ457728 & - & GQ457848 & - & - \\
\hline 88 & Calliophis calligaster & EF 137411 & - & EF137403 & EF 137419 & - & - \\
\hline 89 & Calliophis japonicus & AF217831 & - & - & - & - & - \\
\hline 90 & Calliophis melanurus & KC347458 & KC347351 & KC347502 & KC347391 & - & KC347429 \\
\hline 91 & Calloselasma rhodostoma & AY223562 & AY352718 & AY352813 & - & - & - \\
\hline 92 & Calotes versicolor & AY572870 & JX668217 & JX857560 & JX838985 & DQ340705 & - \\
\hline 93 & Candoia aspera & U69751 & EF545068 & - & - & - & - \\
\hline
\end{tabular}




\begin{tabular}{|c|c|c|c|c|c|c|c|}
\hline 94 & Candoia carinata & AY099984 & EU419850 & _- & AY099961 & FJ433974 & AY988065 \\
\hline 95 & Caraiba andreae & FJ416743 & - & FJ416817 & - & - & - \\
\hline 96 & Carphophis amoenus & AF471067 & AY577022 & - & DQ112082 & - & - \\
\hline 97 & Casarea dussumieri & U69755 & AF544827 & - & AF 544731 & EU402632 & EU402840 \\
\hline 98 & Causus defilippi & GU045452 & GU045452 & AY223617 & - & EU402633 & - \\
\hline 99 & Causus resimus & AY223555 & AY223662 & AY223616 & AF544696 & - & - \\
\hline 100 & Cemophora coccinea & KF216147 & - & DQ902282 & AF471132 & - & - \\
\hline 101 & Cerastes cerastes & AF471028 & HQ267811 & EU624222 & AF544679 & - & EU852329 \\
\hline 102 & Cerberus rynchops & EF395900 & EF395851 & U49327 & EF395925 & - & - \\
\hline 103 & Cerrophidion barbouri & HM363641 & HM363640 & HM363642 & - & - & - \\
\hline 104 & Cerrophidion godmani & AY220328 & DQ305442 & AY220349 & - & - & - \\
\hline 105 & Chamaeleo calyptratus & NC12420 & HF 570443 & HF570566 & HF570667 & GU457847 & - \\
\hline 106 & Charina bottae & AY099986 & AF544816 & AF302959 & AY099971 & FJ433978 & AY988076 \\
\hline 107 & Charina reinhardtii & - & - & AF302943 & - & - & - \\
\hline 108 & Charina trivirgata & - & GQ200595 & AF302944 & - & EU402649 & EU402852 \\
\hline 109 & Charina umbratica & KF811115 & - & AF 302977 & KF811099 & KF811062 & KF811163 \\
\hline 110 & Chilabothrus striatus & - & - & KC329966 & KC329991 & KC330056 & KC330027 \\
\hline 111 & Chilomeniscus cinctus & - & - & U49305 & - & - & - \\
\hline 112 & Chilomeniscus stramineus & GQ895856 & - & - & GQ895800 & - & - \\
\hline 113 & Chionactis occipitalis & GQ895857 & - & - & GQ895801 & - & - \\
\hline 114 & Chironius scurrulus & KX660434 & KX660156 & KX660560 & KX660295 & - & - \\
\hline 115 & Chironius scurrulus & KX660535 & KX660265 & - & - & - & - \\
\hline 116 & Chrysopelea taprobanica & KC347459 & KC347354 & KM673290 & KC347394 & - & KC347432 \\
\hline 117 & Clelia clelia & GQ895859 & AF 158472 & - & JQ598973 & - & - \\
\hline 118 & Clonophis kirtlandii & KF258647 & - & KF258630 & - & KF258596 & - \\
\hline 119 & Coelognathus radiatus & DQ902121 & - & DQ902317 & DQ902079 & - & - \\
\hline 120 & Coluber algirus & - & AY643349 & - & - & - & - \\
\hline 121 & Coluber constrictor priapus & AY486914 & L01770 & AY487041 & AY486938 & EU402634 & EU402841 \\
\hline 122 & Coluber dorri & AY188040 & AY188081 & AY487042 & AY188001 & - & - \\
\hline 123 & Coluber zebrinus & AY188043 & AY188084 & AY487058 & AY188004 & - & - \\
\hline 124 & Compsophis albiventris & - & AY188050 & FJ404351 & AY187972 & - & - \\
\hline 125 & Compsophis boulengeri & EF203995 & EF204007 & - & EF204001 & - & - \\
\hline 126 & Coniophanes fissidens & EF078538 & - & EF078586 & - & - & - \\
\hline 127 & Conophis lineatus & JQ598924 & GU018161 & - & JQ598975 & JQ599016 & - \\
\hline 128 & Contia tenuis & GU112384 & AY577030 & GU112419 & AF471134 & GU112346 & - \\
\hline 129 & Corallus annulatus & KC750012 & - & KC750018 & KC750007 & JX576167 & KC750047 \\
\hline 130 & Crotaphopeltis hotamboeia & AF428023 & - & - & - & - & - \\
\hline 131 & Crotaphopeltis tornieri & AF471093 & - & - & AF471112 & - & - \\
\hline 132 & Cryophis hallbergi & GQ334481 & - & GQ334582 & GQ895807 & - & - \\
\hline 133 & Cubophis cantherigerus & - & - & FJ416818 & AF544694 & FJ433999 & - \\
\hline 134 & Cyclocorinae sp & MG458751 & - & - & MG458760 & - & - \\
\hline 135 & Cyclocorus lineatus & MG458754 & - & - & MG458764 & - & - \\
\hline 136 & Cyclocorus nuchalis & MG458750 & - & - & MG458759 & - & - \\
\hline 137 & Cyclophiops multicinctus & KX660435 & KX660157 & - & KX660296 & - & \\
\hline 138 & Cylindrophis maculatus & KC347460 & KC347355 & KC347494 & KC347395 & - & KC347433 \\
\hline 139 & Cylindrophis ruffus & AB179619 & AB179619 & AB179619 & AF471133 & AY988037 & AY988071 \\
\hline 140 & Daboia russellii & EU913478 & EU913478 & EU913478 & AF471156 & EU402636 & EU402843 \\
\hline 141 & Dasypeltis fasciata & KX660463 & KX660190 & KX660589 & KX660328 & - & - \\
\hline
\end{tabular}




\begin{tabular}{|c|c|c|c|c|c|c|c|}
\hline 142 & Deinagkistrodon acutus & EU913476 & EU913476 & EU913476 & - & - & - \\
\hline 143 & Demansia vestigiata & EU547045 & EU547143 & EU547003 & AY058927 & - & - \\
\hline 144 & Dendrelaphis marenae & KX660515 & KX660245 & - & KX660386 & - & - \\
\hline 145 & Dendrelaphis tristis & KC347462 & КС347359 & KC347493 & KC347398 & - & KC347436 \\
\hline 146 & Dendroaspis angusticeps & - & FJ404194 & JF357927 & AF544735 & FJ433988 & - \\
\hline 147 & Dendroaspis jamesoni & KX660464 & KX660191 & KX660590 & KX660329 & _ & _- \\
\hline 148 & Dendroaspis polylepis & FJ404295 & - & AY058974 & FJ387197 & - & - \\
\hline 149 & Dendrophidion dendrophis & GQ895865 & - & - & GQ895809 & - & - \\
\hline 150 & Dendrophidion percarinatum & KX660556 & KX660291 & KX660667 & KX660431 & - & _- \\
\hline 151 & Dendrophidion percarinatus & - & HM582217 & - & HQ157822 & - & - \\
\hline 152 & Denisonia devisi & EU547071 & EU547169 & EU547023 & EU546933 & - & - \\
\hline 153 & Diadophis punctatus & AF471094 & AF544793 & EU194091 & AF471122 & EU402637 & _- \\
\hline 154 & Dinodon rufozonatum & AF471063 & HM439982 & KF732917 & JF827695 & JQ599018 & - \\
\hline 155 & Dipsadoboa brevirostris & - & KX660285 & KX660661 & KX660424 & - & - \\
\hline 156 & Dipsadoboa duchesnii & KX660453 & KX660180 & - & KX660319 & - & - \\
\hline 157 & Dipsas catesbyi & JQ598926 & Z46496 & - & JQ598977 & JQ599021 & - \\
\hline 158 & Dipsas peruana & KX660538 & KX660269 & - & KX660406 & - & - \\
\hline 159 & Dipsas pratti & GQ334482 & - & GQ334583 & - & - & - \\
\hline 160 & Ditypophis sp. & - & - & & - & JQ073079 & JQ073200 \\
\hline 161 & Drymarchon corais & AF471064 & HM582218 & DQ902314 & AF471137 & - & - \\
\hline 162 & Drymobius rhombifer & GQ927320 & HM582220 & - & GQ927313 & - & - \\
\hline 163 & Drymoluber dichrous & GQ895869 & HM582221 & - & HQ157824 & - & - \\
\hline 164 & Dryophiops philippina & KX660517 & KX660247 & KX660641 & KX660388 & - & - \\
\hline 165 & Echinanthera undulata & JQ598929 & JQ598870 & - & JQ598978 & JQ599022 & - \\
\hline 166 & Echiopsis atriceps & EU547080 & EU547178 & EU547032 & EU546942 & - & - \\
\hline 167 & Echiopsis curta & EU547072 & EU547170 & EU547024 & EU546934 & - & - \\
\hline 168 & Echis carinatus & GQ359436 & GQ359685 & GQ359524 & - & - & EU852325 \\
\hline 169 & Eirenis modestus & AY486933 & AY376792 & AY487072 & AY486957 & - & - \\
\hline 170 & Elaphe bella & DQ902134 & - & DQ902316 & DQ902097 & - & - \\
\hline 171 & Elaphe poryphyracea & - & GQ181130 & GQ181130 & - & - & - \\
\hline 172 & Elaphe quatuorlineata & AY486931 & AF215267 & AY487067 & AY486955 & - & - \\
\hline 173 & Elapognathus coronata & EU547069 & EU547167 & EU547021 & EU546931 & - & - \\
\hline 174 & Elapomorphus quinquelineatus & JQ598930 & GQ457735 & - & GQ457855 & JQ599023 & - \\
\hline 175 & Elapsoidea nigra & AF217820 & - & AY058975 & AY058930 & - & - \\
\hline 176 & Elapsoidea semiannulata & AF039260 & JF357946 & JF357928 & AF544678 & FJ433987 & - \\
\hline 177 & Elgaria multicarinata & AF361528 & AY649151 & DQ364660 & AF039479 & GU457854 & GU457977 \\
\hline 178 & Emydocephalus annulatus & DQ233940 & DQ234001 & FJ593196 & FJ587172 & - & - \\
\hline 179 & Enhydris dussumieri & JX463014 & JX463016 & - & JX463012 & - & - \\
\hline 180 & Enhydris enhydris & EF395904 & EF395855 & GU997190 & EF395929 & - & _- \\
\hline 181 & Enhydris plumbea & EF395910 & EF395861 & U49328 & EF395934 & - & - \\
\hline 182 & Enulius $s p$. & GQ895870 & _ & - & GQ895813 & - & - \\
\hline 183 & Ephalophis greyae & JX002976 & FJ587208 & FJ593197 & FJ587173 & - & - \\
\hline 184 & Epicrates cenchria & HQ399501 & _- & KC329975 & KC330008 & KC330073 & - \\
\hline 185 & Epicrates striatus & U69791 & - & - & - & - & - \\
\hline 186 & Eristicophis macmahoni & AJ275711 & EU624293 & EU624227 & - & - & - \\
\hline 187 & Erythrolamprus aesculapii & GQ895871 & GQ457736 & - & GQ895814 & JQ599024 & - \\
\hline 188 & Eryx colubrinus & U69811 & AF544819 & - & AF544716 & EU402639 & DQ465571 \\
\hline 189 & Eunectes notaeus & HQ399499 & AM236347 & KC329978 & HQ399536 & KC330076 & HQ399516 \\
\hline
\end{tabular}




\begin{tabular}{|c|c|c|c|c|c|c|c|}
\hline 190 & Euprepiophis mandarinus & DQ902115 & - & DQ902294 & DQ902073 & - & - \\
\hline 191 & Euprepiophis perlacea & KF669239 & KF850472 & KF850472 & - & - & - \\
\hline 192 & Exallodontophis albignaci & EU394724 & - & - & - & - & - \\
\hline 193 & Exiliboa placata & AY099989 & AF512742 & - & AY099973 & EU402640 & AY988068 \\
\hline 194 & Farancia abacura & U69832 & Z46491 & U49307 & AF471141 & - & KR814740 \\
\hline 195 & Furina diadema & EU547053 & EU547151 & EU547008 & EU546917 & - & - \\
\hline 196 & Furina ornata & EU547054 & EU547152 & EU547009 & EU546918 & - & - \\
\hline 197 & Geophis carinosus & GQ895872 & - & - & GQ895815 & - & - \\
\hline 198 & Geophis godmani & JQ598932 & JQ598877 & - & - & JQ599026 & - \\
\hline 199 & Gloydius brevicaudus & JQ687496 & - & JQ687477 & JQ687515 & - & - \\
\hline 200 & Gloydius halys & AY223564 & AF 057238 & JQ356856 & - & - & - \\
\hline 201 & Gomesophis brasiliensis & - & GQ457737 & - & - & - & - \\
\hline 202 & Gonionotophis capensis & - & AF544798 & - & AF544703 & FJ433995 & - \\
\hline 203 & Gonyosoma oxycephalum & AF471084 & Z46490 & DQ902309 & KC010302 & - & - \\
\hline 204 & Grayia ornata & - & AF158503 & AF544663 & AF544684 & FJ434002 & - \\
\hline 205 & Hapsidophrys smaragdina & AY612057 & AY611875 & - & DQ112078 & FJ434003 & - \\
\hline 206 & Hebius craspedogaster & - & - & - & KJ685622 & - & - \\
\hline 207 & Hebius sauteri & - & - & - & KJ685651 & - & - \\
\hline 208 & Helicops angulatus & AF471037 & GQ457738 & - & AF 471160 & JQ599027 & - \\
\hline 209 & Helicops infrataeniatus & JQ598933 & GQ457740 & - & GQ457859 & - & - \\
\hline 210 & Helicops pictiventris & - & - & U49310 & - & - & - \\
\hline 211 & Heloderma suspectum & NC8776 & NC_8776 & NC_8776 & AY487348 & GU457856 & GU457979 \\
\hline 212 & Hemachatus haemachatus & AF217821 & - & - & - & - & - \\
\hline 213 & Hemerophis socotrae & AY188042 & AY188083 & AY487055 & AY188003 & - & - \\
\hline 214 & Hemiaspis damelii & EU547073 & DQ233979 & FJ593193 & FJ587161 & - & - \\
\hline 215 & Hemiaspis signata & EU547074 & DQ233980 & EU547026 & EU546936 & - & - \\
\hline 216 & Hemorrhois algirus & AY486911 & - & AY487037 & AY486935 & - & - \\
\hline 217 & Herpetoreas platyceps & KJ685690 & - & - & KJ685640 & - & - \\
\hline 218 & Heterodon platirhinos & GU112412 & AY577028 & AF402659 & JQ598986 & JQ599028 & - \\
\hline 219 & Hierophis jugularis & AY486917 & AY376769 & AY487046 & AY486941 & - & - \\
\hline 220 & Hierophis spinalis & AY486924 & AY376773 & AY487056 & AY486948 & - & - \\
\hline 221 & Hologerrhum philippinum & MG458758 & - & - & MG458766 & - & - \\
\hline 222 & Homoroselaps lacteus & AY611992 & AY611809 & FJ404338 & AY611901 & JQ599029 & - \\
\hline 223 & Hoplocephalus bitorquatus & EU547079 & EU547177 & EU547031 & EU546941 & - & - \\
\hline 224 & Hormonotus modestus & FJ404296 & FJ404195 & FJ404360 & FJ404261 & - & - \\
\hline 225 & Hydrelaps darwiniensis & KC014413 & DQ234046 & FJ593200 & FJ587175 & - & - \\
\hline 226 & Hydrodynastes bicinctus & JQ598935 & GQ457742 & - & GQ457862 & JQ599030 & - \\
\hline 227 & Hydromorphus concolor & GQ895874 & - & - & GQ895817 & - & - \\
\hline 228 & Hydrophis lamberti & KC014421 & KC014345 & KC014496 & KC014300 & - & - \\
\hline 229 & Hydrops triangularis & - & GQ457744 & - & AF471158 & JQ599032 & - \\
\hline 230 & Hypnale zara & KC347463 & KC347363 & KC347513 & KC347402 & - & KC347440 \\
\hline 231 & Hypsiglena affinis & - & - & EU363055 & - & - & - \\
\hline 232 & Hypsiglena chlorophaea & KJ486459 & KF548588 & EU728577 & - & - & - \\
\hline 233 & Hypsiglena torquata & AF471038 & - & GQ334584 & AF471159 & - & - \\
\hline 234 & Hypsirhynchus ferox & GQ895875 & AF158515 & FJ416816 & GQ895818 & - & - \\
\hline 235 & Hypsirhynchus parvifrons & FJ416740 & - & FJ416814 & - & - & - \\
\hline 236 & Ialtris dorsalis & FJ416735 & AF 158525 & FJ416809 & - & - & - \\
\hline 237 & Imantodes cenchoa & GQ334484 & GQ457745 & GQ334589 & GQ457865 & EU402643 & EU402847 \\
\hline
\end{tabular}




\begin{tabular}{|c|c|c|c|c|c|c|c|}
\hline 238 & Indotyphlops braminus & DQ343649 & - & - & AF544717 & FJ433959 & - \\
\hline 239 & Lachesis stenophrys & AY223603 & AF057267 & U96026 & - & EU402644 & - \\
\hline 240 & Lampropeltis getula & AF337153 & _- & DQ360485 & - & - & - \\
\hline 241 & Lampropeltis ruthveni & AF337064 & - & AY739641 & FJ627803 & - & - \\
\hline 242 & Lamprophis fuliginosus & AF471060 & AY188079 & FJ404365 & FJ404270 & EU402646 & EU402849 \\
\hline 243 & Langaha madagascariensis & - & AY188059 & FJ404370 & AY187981 & - & - \\
\hline 244 & Laticauda colubrina & EU547040 & EU547138 & FJ606513 & EU366446 & FJ433990 & - \\
\hline 245 & Laticauda frontalis & - & FJ587206 & FJ593190 & FJ587157 & - & - \\
\hline 246 & Laticauda guineai & - & - & FJ606516 & & - & - \\
\hline 247 & Laticauda laticaudata & FJ587153 & FJ587204 & FJ593192 & FJ587159 & - & - \\
\hline 248 & Laticauda saintgironsi & - & - & FJ606506 & & - & - \\
\hline 249 & Laticauda semifasciata & AB701339 & - & - & - & - & - \\
\hline 250 & Leiopython albertisii & - & EF545053 & - & - & - & - \\
\hline 251 & Leptodeira annulata & GQ334493 & GQ457746 & GQ334594 & AF544690 & FJ433998 & - \\
\hline 252 & Leptophis depressirostris & KX660465 & - & KX660592 & KX660331 & - & - \\
\hline 253 & Leptophis diplotropis & KX660540 & KX660271 & - & KX660408 & - & - \\
\hline 254 & Leptotyphlops conjunctus & - & GQ469280 & - & GQ469069 & GQ469184 & - \\
\hline 255 & Leptotyphlops humilis & - & - & & - & EU402648 & EU402851 \\
\hline 256 & Lepturophis albofuscus & KX660500 & KX660231 & KX660629 & KX660372 & - & - \\
\hline 257 & Liasis albertisii & U69835 & - & - & - & - & - \\
\hline 258 & Liasis childreni & U69837 & - & - & - & - & - \\
\hline 259 & Liasis mackloti & U69839 & EF545051 & - & AF544726 & FJ433970 & - \\
\hline 260 & Lichanura trivirgata & U69844 & - & - & AF544687 & - & - \\
\hline 261 & Liolaemus darwinii & KC150152 & - & DQ237768 & $\mathrm{JF} 272870$ & KP820851 & KP820640 \\
\hline 262 & Liopeltis frenatus & KX660457 & KX660184 & KX660583 & KX660323 & - & - \\
\hline 263 & Liophidium chabaudi & EU394721 & FJ404210 & FJ404372 & FJ387210 & - & - \\
\hline 264 & Liophis amarali & - & GQ457747 & - & GQ457867 & - & - \\
\hline 265 & Liophis lineatus & - & - & - & DQ469789 & DQ469795 & DQ469791 \\
\hline 266 & Liophis meridionalis & - & GQ457750 & - & GQ457870 & - & - \\
\hline 267 & Liopholidophis sexlineatus & DQ979985 & AY188063 & FJ404373 & AY187985 & - & - \\
\hline 268 & Liotyphlops albirostris & AF544672 & AF366762 & - & AF544727 & EU402650 & EU402853 \\
\hline 269 & Loxocemus bicolor & AY099993 & AF544828 & - & AY444035 & EU402651 & - \\
\hline 270 & Lycodonomorphus whytii & FJ404300 & FJ404200 & FJ404375 & FJ387201 & - & - \\
\hline 271 & Lycodryas citrinus & - & - & HE798413 & - & JQ073076 & JQ073197 \\
\hline 272 & Lystrophis histricus & - & GQ457753 & - & - & - & - \\
\hline 273 & Lytorhynchus diadema & DQ112076 & HQ267794 & - & DQ112079 & - & - \\
\hline 274 & Macrelaps microlepidotus & AY611993 & AY611810 & FJ404340 & AY611902 & - & - \\
\hline 275 & Macropisthodon rhodomelas & KX660528 & KX660258 & JQ687427 & KX660399 & - & - \\
\hline 276 & Macropisthodon rudis & JQ687434 & - & AY487064 & JQ687452 & - & - \\
\hline 277 & Macroprotodon cucullatus & AF471087 & AY188065 & - & AF471145 & - & - \\
\hline 278 & Macrovipera lebetina & AJ275713 & EU624294 & DQ897729 & - & - & - \\
\hline 279 & Magliophis exiguum & AF471071 & - & FJ416798 & AF471117 & - & - \\
\hline 280 & Malayopython reticulatus & U69860 & EF545062 & - & AF544675 & FJ433969 & EU624119 \\
\hline 281 & Malpolon moilensis & DQ486333 & HQ267802 & DQ486309 & DQ486157 & - & - \\
\hline 282 & Manolepis putnami & GQ895878 & GU018171 & - & GQ895820 & JQ599035 & _- \\
\hline 283 & Mastigodryas bifossatus & - & HM582223 & - & HQ 157827 & - & - \\
\hline 284 & Mastigodryas boddaerti & GQ895867 & HM582224 & - & GQ895811 & - & - \\
\hline 285 & Mastigodryas melanolomus & GQ895868 & - & - & - & - & - \\
\hline
\end{tabular}


HQ207116 HQ207158

Microcephalophis gracilis

Micropechis ikaheka

Micruroides euryxanthus

Micrurus fulvius

Micrurus surinamensis

Mitophis asbolepis

Montivipera wagneri

Morelia boeleni

Morelia bredli

Morelia carinata

Morelia viridis

Myersophis alpestris

Myriopholis adleri

Naja haje arabica

Naja kaouthia

Namibiana occidentalis

Natriciteres olivacea

Natrix natrix

Neelaps calonotus

Nerodia erythrogaster

Nerodia fasciata

Ninia atrata

Ninia sebae

Notechis ater

Notechis scutatus

Oligodon arnensis

Oligodon ningshaanensis

Oligodon theobaldi

Oocatochus rufodorsatus

Ophiophagus hannah

Ophryacus undulatus

Opisthotropis cheni

Oreocryptophis porphyraceus

Orthriophis hodgsonii

Orthriophis taeniurus taeniura

Ovophis chasen

Ovophis okinavensis

Oxybelis aeneus

Oxyrhabdium leporinum

Oxyrhopus fitzingeri

Oxyrhopus guibei

Oxyrhopus trigeminus

Oxyuranus scutellatus

Pantherophis guttatus

Parahydrophis mertoni

- AY701024 _ _

DQ486349 _ _

KC014420 KC014341 KC014494

EU547042 FJ587207 GQ397208

EF137416 Z46483 EF137408

U69846 GU045453 GU045453

EF137415 AF544799 JF308709

GQ469086 GQ469210

DQ486173

KC014299

EU366449

EF137423

AY058935 EU402653 EU402856

AF544708 FJ433991

GQ469059 GQ469174

- _ _ JN870213 _

EF545047

KJ666638 EF545043 -

EF545095 EF545044

EF545098 EF545048

MG458752 GQ469246

MG458762

$$
\text { - } \quad \text { GQ469246 _ }
$$

GQ359500 GQ359749 GQ387074

FR693728 GQ359757 EU624209

- $\quad$ GQ469251

AY058938 EU402654 EU402857

AF471058 AF544801 .

GQ469074 GQ469189

AY487749

AY487794

AF471146

EU547060 EU547158 EF210841

AF544697 EU402655 EU402858

$\begin{array}{llll} & & \end{array}$

$\begin{array}{lll}\text { AF402912 - } & \text { AF420084 JN090137 }\end{array}$

AF402910 - AY873705 -

KF258597

JQ598937 JQ598882 GQ334659

GQ895879

EU547082 EU547180 EU547034$$
-
$$$$
\text { - } \quad-\quad \text { - }
$$

KC347464 KC347365 KC347504 KC347404

EU402656 EU402859

KJ638715 NC_26083 KJ719252 KJ638717

- $\quad$ HM591515 _

KC990020 KC990020 DQ902301

EU921899 JN687931 EU921899 AY058940

AY223586 AF057256 AY223633

GQ281779 _-

JQ687416

KF669255

DQ902136 _

DQ902318

EF076709 HM439981 DQ902305

AY352760 AY352729 AY352825

AB175670 AB175670 AB175670

AF471056 HM582225 _

AF471148

AF471029 _

DQ112081

KX660541 KX660272 -

KX660409

JQ598938 JQ627289

JQ598989 JQ599038

- KX660274 KX660653

EU547051 EU547149 EF210827 EU546916

AM236349 AM236349 AM236349 DQ902070

KC014451 DQ234048 FJ593201 FJ587177 


\begin{tabular}{|c|c|c|c|c|c|c|}
\hline 334 & Parastenophis betsileanus & GU994802 & GU994852 & - & GU994827 & - \\
\hline 335 & Pareas boulengeri & JF827683 & _- & - & JF827710 & - \\
\hline 336 & Pareas carinatus & JF827677 & AF544802 & JF827653 & JF827702 & FJ433985 \\
\hline 337 & Pareas formosensis & HQ528535 & - & HQ528434 & KJ642213 & - \\
\hline 338 & Pareas hamptoni & KX694896 & KX694656 & - & - & KX694746 \\
\hline 339 & Pareas iwasakii & KJ642160 & - & - & KJ642209 & - \\
\hline 340 & Pareas margaritophorus & AY425805 & - & - & KJ642217 & - \\
\hline 341 & Pareas monticola & JF827689 & - & - & JF827715 & - \\
\hline 342 & Pareas nuchalis & - & - & U49311 & - & - \\
\hline 343 & Pareas stanleyi & JN230704 & - & JN230705 & JN230703 & - \\
\hline 344 & Phalotris lemniscatus & JQ598941 & GQ457756 & & GQ457877 & JQ599039 \\
\hline 345 & Philodryas chamissonis & - & - & HM639951 & - & - \\
\hline 346 & Philothamnus natalensis & KX660555 & KX660290 & KX660666 & KX660430 & - \\
\hline 347 & Philothamnus natalensis2 & AY612069 & AY611887 & - & & - \\
\hline 348 & Phyllorhynchus decurtatus & AF471083 & - & - & AF544728 & FJ434004 \\
\hline 349 & Pituophis catenifer & - & - & AF141106 & FJ627790 & - \\
\hline 350 & Pituophis lineaticollis & - & AF512746 & JF308340 & FJ627804 & - \\
\hline 351 & Pituophis melanoleucus & AF337112 & - & - & - & - \\
\hline 352 & Plagiopholis styani & EU496918 & - & - & EU496916 & - \\
\hline 353 & Platyceps karelini & AY486918 & - & - & AY486942 & - \\
\hline 354 & Platyceps rogersi & - & AY188082 & AY487052 & - & - \\
\hline 355 & Pliocercus elapoides & GQ895882 & - & - & GQ895824 & - \\
\hline 356 & Pliocercus euryzona & KX660440 & KX660165 & KX660568 & KX660304 & - \\
\hline 357 & Porthidium yucatanicum & DQ061215 & JN870198 & DQ061244 & - & - \\
\hline 358 & Proatheris superciliaris & AJ275685 & EU624296 & EU624230 & - & - \\
\hline 359 & Prosymna janii & FJ404319 & FJ404222 & FJ404389 & FJ404293 & - \\
\hline 360 & Protobothrops mangshanensis & HM567537 & AY352726 & HM567469 & JQ687524 & - \\
\hline 361 & Psammodynastes pictus & KX660507 & KX660237 & - & KX660378 & - \\
\hline 362 & Psammodynastes pulverulentus & AF471031 & AF544813 & - & AF471157 & - \\
\hline 363 & Psammodynastes sp. & FJ404318 & FJ404221 & FJ404383 & FJ387218 & - \\
\hline 364 & Psammophis schokari & AY612034 & AY611852 & EF128005 & AY611943 & - \\
\hline 365 & Psammophis sp. & DQ486444 & - & DQ486280 & DQ486184 & - \\
\hline 366 & Pseudalsophis dorsalisial & JQ598946 & JQ598892 & - & JQ598994 & - \\
\hline 367 & Pseudalsophis elegans & - & - & - & - & JQ599042 \\
\hline 368 & Pseudaspis cana & AY612080 & AY611898 & DQ486319 & DQ486167 & - \\
\hline 369 & Pseudechis australis & EU547046 & EU547144 & AY340174 & EU546912 & - \\
\hline 370 & Pseudechis porphyriacus & EU547047 & EU547145 & AY340170 & EU546913 & - \\
\hline 371 & Pseudoboa nigra & JQ598948 & GQ457764 & - & AF544729 & JQ599043 \\
\hline 372 & Pseudocerastes fieldi & AJ275716 & AJ275769 & - & - & - \\
\hline 373 & Pseudoeryx plicatilis & GQ895885 & GQ457765 & - & GQ895826 & - \\
\hline 374 & Pseudoficimia frontalis & GQ895886 & - & - & GQ895827 & - \\
\hline 375 & Pseudoleptodeira latifasciata & EU728579 & EU728579 & GQ334661 & - & - \\
\hline 376 & Pseudonaja modesta & EU547049 & EU547147 & DQ098492 & EU546915 & - \\
\hline 377 & Pseudorabdion longiceps & KX660529 & KX660259 & - & KX660400 & - \\
\hline 378 & Pseudotomodon trigonatus & - & GQ457766 & - & GQ457887 & - \\
\hline 379 & Pseudoxenodon karlschmidti & AF471080 & JF697330 & - & AF471102 & JQ599045 \\
\hline 380 & Pseustes poecilonotus & KX660436 & KX660160 & KX660563 & KX660299 & - \\
\hline 381 & Pseustes poecilonotus2 & KF669676 & - & KF669693 & KF669710 & - \\
\hline
\end{tabular}




\begin{tabular}{|c|c|c|c|c|c|c|c|}
\hline 382 & Pseustes poecilonotus3 & KF669671 & _- & KF669687 & KF669705 & - & - \\
\hline 383 & Psomophis joberti & GQ895887 & GQ457768 & - & GQ895828 & JQ599046 & _- \\
\hline 384 & Ptyas mucosus & AF471054 & KC589121 & AY487063 & GQ225670 & - & - \\
\hline 385 & Ptychophis flavovirgatus & - & GQ457769 & - & GQ457890 & - & - \\
\hline 386 & Python bivittatus & JX401131 & KF010492 & _- & AF435016 & XM7433022 & _- \\
\hline 387 & Pythonodipsas carinata & - & AY188075 & FJ404386 & AY187997 & - & - \\
\hline 388 & Ramphotyphlops acuticauda & JQ910543 & - & - & - & GU902381 & - \\
\hline 389 & Ramphotyphlops australis & JQ910537 & AY442843 & - & AF039474 & JQ910312 & _- \\
\hline 390 & Ramphotyphlops braminus & DQ343649 & DQ343649 & DQ343649 & _- & _- & AY662612 \\
\hline 391 & Regina grahami & AF402918 & - & KF258633 & - & KF258599 & - \\
\hline 392 & Regina rigida & AF471052 & _- & KF258642 & AF471120 & KF258608 & - \\
\hline 393 & Regina septemvittata & AF402917 & - & KF258629 & - & KF258595 & - \\
\hline 394 & Rena humilis & AY099991 & AB079597 & AB079597 & AY099979 & - & - \\
\hline 395 & Rhabdophis nuchalis & GQ281786 & - & JQ687413 & KF800925 & - & - \\
\hline 396 & Rhabdophis subminiatus & GQ281777 & AF544805 & JQ687411 & AF544713 & JQ599047 & _- \\
\hline 397 & Rhadinaea flavilata & AF471078 & - & - & AF471152 & - & - \\
\hline 398 & Rhamphiophis oxyrhynchus & JQ598953 & FJ404213 & _- & AF544710 & JQ599049 & - \\
\hline 399 & Rhinobothryum lentiginosum & - & HM582227 & - & AF544693 & - & - \\
\hline 400 & Rhinocheilus lecontei & AF337109 & - & AF138773 & FJ627788 & - & - \\
\hline 401 & Rhinoleptus sp. & - & GQ469242 & - & GQ469078 & GQ469193 & - \\
\hline 402 & Rhinophis drummondhayi & AF544673 & AY701028 & - & AF544719 & FJ433966 & - \\
\hline 403 & Rhinophis erangaviraji & KC347490 & KC347371 & KC347503 & KC347410 & - & KC347448 \\
\hline 404 & Rhinoplocephalus bicolor & EU547068 & EU547166 & EU547020 & EU546930 & _- & _- \\
\hline 405 & Rhinoplocephalus nigrescens & EU547070 & EU547168 & EU547022 & EU546932 & - & - \\
\hline 406 & Rhinotyphlops episcopus & - & - & - & - & KC848449 & - \\
\hline 407 & Rhinotyphlops lalandei & - & - & - & - & GU902386 & - \\
\hline 408 & Sanzinia madagascariensis & U69866 & AY336066 & - & EU403580 & AY988033 & AY988067 \\
\hline 409 & Scaphiodontophis annulatus & GQ927323 & - & - & GQ927318 & - & - \\
\hline 410 & Scaphiophis albopunctatus & DQ486345 & - & DQ486321 & DQ486169 & _- & - \\
\hline 411 & Senticolis triaspis & DQ902127 & _- & AF138775 & DQ902086 & - & - \\
\hline 412 & Siagonodon septemstriatus & - & GQ469232 & - & GQ469076 & GQ469191 & - \\
\hline 413 & Sibon annulatus & KX660443 & KX660169 & KX660572 & KX660308 & - & - \\
\hline 414 & Sibon nebulatus & EU728583 & AF544806 & GQ334662 & AF544736 & - & - \\
\hline 415 & Sibon sartorrii & EF078540 & - & EF078588 & - & - & - \\
\hline 416 & Sibynomorphus garmani & - & - & _- & GQ457891 & - & _- \\
\hline 417 & Sibynomorphus mikaniirial & JQ598954 & JQ627298 & _- & - & JQ599050 & _- \\
\hline 418 & Sibynomorphus turgidus & KX660547 & KX660279 & KX660659 & KX660418 & - & - \\
\hline 419 & Sibynophis subpunctatus & KC347471 & KC347373 & KC347516 & KC347411 & - & KC347449 \\
\hline 420 & Simalia boeleni & KJ666597 & _- & _- & KF811106 & KF811070 & - \\
\hline 421 & Simoselaps anomalus & EU547061 & EU547159 & EU547014 & EU546924 & - & KF811168 \\
\hline 422 & Sinomicrurus japonicus & - & - & AY058971 & AY058926 & - & - \\
\hline 423 & Sinonatrix annularis & JQ687431 & HM439988 & JQ687424 & AF544712 & - & - \\
\hline 424 & Siphlophis cervinus & GQ895888 & AF 158536 & - & GQ895829 & - & - \\
\hline 425 & Sistrurus catenatus & AY223610 & AF259119 & - & KF410311 & - & - \\
\hline 426 & Sordellina punctata & JQ598956 & JQ598903 & - & JQ599000 & JQ599052 & _ \\
\hline 427 & Spilotes pullatus & AF471041 & HM582228 & KF669677 & AF471110 & - & - \\
\hline 428 & Spilotes sulphureus & KX660545 & KX660276 & KX660656 & KX660415 & - & - \\
\hline 429 & Stenophis betsileanus & - & - & FJ404387 & - & - & - \\
\hline
\end{tabular}




\begin{tabular}{|c|c|c|c|c|c|c|c|}
\hline 430 & Stenophis citrinus & AY612047 & AY611865 & KF258627 & AY611956 & - & - \\
\hline 431 & Stenorrhina freminvillei & GQ895889 & - & - & GQ895830 & - & - \\
\hline 432 & Stoliczkia borneensis & - & AF544808 & - & AF544721 & FJ433982 & - \\
\hline 433 & Storeria dekayi & AF471050 & JQ598904 & - & AF471154 & KF258593 & - \\
\hline 434 & Suta fasciata & EU547064 & EU547162 & EU547016 & EU546927 & - & - \\
\hline 435 & Suta monachus & EU547067 & EU547165 & EU547019 & EU546929 & - & - \\
\hline 436 & Suta spectabilis & EU547065 & EU547163 & EU547017 & EU546928 & _- & - \\
\hline 437 & Suta suta & EU547066 & EU547164 & EU547018 & EU366452 & - & - \\
\hline 438 & Sympholis lippiens & GQ895890 & - & - & GQ895831 & - & - \\
\hline 439 & Tachymenis peruviana & - & GQ457774 & - & GQ457895 & JQ599054 & - \\
\hline 440 & Taeniophallus affinis & JQ598957 & GQ457733 & - & GQ457853 & JQ599055 & - \\
\hline 441 & Tantalophis discolor & EF078541 & - & EF078589 & - & - & - \\
\hline 442 & Tantilla melanocephala & - & AF 158491 & - & - & - & - \\
\hline 443 & Tantilla relicta & AF471045 & - & - & AF471107 & - & - \\
\hline 444 & Telescopus beetzi & KX660551 & KX660287 & KX660663 & KX660426 & - & - \\
\hline 445 & Telescopus fallax & AF471043 & AY188078 & - & AF471108 & - & - \\
\hline 446 & Thamnodynastes hypoconia & KX660523 & KX660253 & KX660643 & KX660394 & - & - \\
\hline 447 & Thamnodynastes pallidus & GQ895891 & GU018166 & - & GQ895832 & - & - \\
\hline 448 & Thamnodynastes strigatus & - & KX660281 & - & KX660420 & - & - \\
\hline 449 & Thamnophis butleri & AF402923 & - & KF258628 & - & KF258594 & - \\
\hline 450 & Thamnophis godmani & - & - & AF420138 & AF471165 & - & - \\
\hline 451 & Thamnophis sirtalis & - & - & - & - & - & XM014075835 \\
\hline 452 & Thelotornis capensis & AF471042 & - & - & AF471109 & - & - \\
\hline 453 & Thelotornis kirtlandii & KX660466 & KX660193 & KX660593 & KX660332 & - & - \\
\hline 454 & Thermophis zhaoermii & GQ166168 & GQ166168 & EU864150 & KF514882 & - & - \\
\hline 455 & Thrasops flavigularis & - & KX660286 & KX660662 & KX660425 & - & - \\
\hline 456 & Thrasops jacksonii & AF471044 & - & - & DQ112084 & - & - \\
\hline 457 & Tomodon dorsatus & GQ895892 & GQ457777 & - & GQ895833 & JQ599059 & - \\
\hline 458 & Toxicocalamus loriae & GQ397170 & GQ397235 & GQ397211 & GQ397225 & - & - \\
\hline 459 & Toxicocalamus preussi & EU547043 & EU547141 & EU547001 & EU546909 & - & - \\
\hline 460 & Toxicodryas blandingii & KX660549 & KX660283 & - & KX660422 & - & - \\
\hline 461 & Toxicodryas pulverulenta & KX660460 & KX660187 & - & AF471118.2 & - & - \\
\hline 462 & Toxicodryas pulverulenta & AF471047 & - & KX660586 & - & - & - \\
\hline 463 & Trachischium monticola & JQ687435 & - & JQ687428 & JQ687453 & - & - \\
\hline 464 & Trimeresurus albolabris & KF311102 & KF311102 & AY352837 & - & - & - \\
\hline 465 & Trimeresurus nebularis & KX660506 & KX660236 & KX660634 & KX660377 & - & - \\
\hline 466 & Trimeresurus trigonocephalus & KC347479 & KC347374 & AY059597 & KC347412 & - & - \\
\hline 467 & Trimorphodon biscutatus & GQ927324 & - & DQ497506 & GQ927319 & EU402662 & EU402864 \\
\hline 468 & Tropidechis carinatus & EU547081 & EU547179 & EU547033 & EU546943 & - & - \\
\hline 469 & Tropidoclonion lineatum & AF402931 & - & KF258638 & _- & KF258604 & - \\
\hline 470 & Tropidodipsas fischeri & KX660553 & KX660289 & KX660664 & KX660428 & - & - \\
\hline 471 & Tropidodryas striaticeps & AF236811 & GQ457778 & - & - & JQ599060 & - \\
\hline 472 & Tropidolaemus subannulatus & KX660525 & KX660255 & KX660644 & KX660396 & - & - \\
\hline 473 & Tropidolaemus wagleri & GQ428472 & AY352727 & AY352822 & - & - & - \\
\hline 474 & Tropidophis feicki & KF811124 & AF512733 & _- & KF811110 & KF811074 & - \\
\hline 475 & Tropidophis wrighti & - & Z46476 & - & - & - & - \\
\hline 476 & Tropidurus plica & EF616028 & EF615664 & EF616320 & EF615737 & JF806028 & JF806213 \\
\hline 477 & Typhlops andasibensis & - & - & - & - & GU902453 & JQ073249 \\
\hline
\end{tabular}




\begin{tabular}{|c|c|c|c|c|c|c|c|}
\hline 478 & Typhlops diardii & - & - & - & - & KF992877 & - \\
\hline 479 & Typhlops jamaicensis & KF993259 & AF366764 & _- & AF544733 & EU402664 & EU402866 \\
\hline 480 & Typhlops luzonensis & - & AF366763 & - & - & GU902393 & - \\
\hline 481 & Typhlops mirus & AM236345 & AM236345 & AM236345 & _- & GU902394 & _- \\
\hline 482 & Typhlops reticulatus & EU747730 & EU747730 & EU747730 & - & GU902396 & - \\
\hline 483 & Typhlops ruber & - & AF512728 & - & - & - & - \\
\hline 484 & Typhlops sulcatus & KF993280 & AF366771 & - & - & GU902441 & - \\
\hline 485 & Typhlops vermicularis & JQ910544 & - & - & - & GU902397 & - \\
\hline 486 & Ungaliophis continentalis & U69870 & AF544833 & - & AF544724 & EU402665 & EU402867 \\
\hline 487 & Ungaliophis panamensis & KX660445 & KX660171 & KX660574 & KX660310 & - & - \\
\hline 488 & Uromacer catesbyi & FJ416714 & AF 158523 & FJ416788 & - & - & - \\
\hline 489 & Varanus salvator & EU621812 & EU621807 & AY033776 & AF435017 & EU402618 & EU402828 \\
\hline 490 & Vipera ammodytes & DQ186504 & EU624297 & EU624232 & - & - & - \\
\hline 491 & Vipera wagneri & AJ275725 & AJ275778 & - & - & - & - \\
\hline 492 & Virginia striatula & KF258657 & - & KF258640 & - & KF258606 & - \\
\hline 493 & Virginia valeriae & KF258656 & - & KF258639 & KP765645 & KF258605 & - \\
\hline 494 & Walterinnesia aegyptia & - & HQ267785 & AY058988 & AY058943 & - & - \\
\hline 495 & Xylophis captaini & $x x x$ & $x x x$ & $x x x$ & $x x x$ & $x x x$ & - \\
\hline 496 & Xylophis perroteti & - & $x x x$ & - & $x x x$ & $x x x$ & $x x x$ \\
\hline 497 & Xylophis stenorhynchus & - & $x x x$ & - & $x x x$ & $x x x$ & - \\
\hline 498 & Xenochrophis asperrimus & - & - & - & - & - & KC347451 \\
\hline 499 & Xenochrophis flavipunctatus & - & AF544809 & - & AF544714 & FJ434001 & - \\
\hline 500 & Xenodermus javanicus & - & AF544810 & U49320 & AF544711 & EU402667 & EU402869 \\
\hline 501 & Xenodon histricus & JQ598962 & - & - & - & JQ599061 & - \\
\hline 502 & Xenopeltis unicolor & AB179620 & AB179620 & AB179620 & AF544689 & EU402668 & DQ465564 \\
\hline 503 & Xenophidion schaeferi & AY574279 & - & - & - & - & - \\
\hline 504 & Xenopholis scalaris & GQ895897 & GU018164 & - & GQ895837 & - & - \\
\hline 505 & Xenotyphlops grandidieri & KF770844 & - & - & - & GU902457 & - \\
\hline 506 & Xenoxybelis argenteus & JQ598944 & GQ457780 & - & GQ457899 & JQ599040 & - \\
\hline 507 & Zamenis hohenackeri & DQ902137 & _ & DQ902320 & DQ902098 & _ & _ \\
\hline
\end{tabular}


Table S2. Partitions and models of sequence evolution used in the Maximum Likelihood (ML) phylogenetic analyses.

\begin{tabular}{|c|c|c|c|}
\hline Subset & Best Model & \# sites & Partition names \\
\hline 1 & $\mathrm{GTR}+\mathrm{I}+\mathrm{G}$ & 582 & $16 s$ \\
\hline 2 & $K 80+1+G$ & 528 & $b d n f 1^{\text {st }}, \operatorname{rag} 12^{\text {nd }}$ \\
\hline 3 & $\mathrm{~K} 80+\mathrm{I}$ & 233 & $b d n f 2^{\text {nd }}$ \\
\hline 4 & $K 80+1+G$ & 233 & $b d n f 3^{\text {rd }}$ \\
\hline 5 & $G T R+1+G$ & 233 & $n d 41^{\text {st }}$ \\
\hline 6 & $G T R+1+G$ & 600 & $n d 42^{\text {nd }}$, cytb $2^{\text {nd }}$ \\
\hline 7 & $\mathrm{GTR}+\mathrm{G}$ & 233 & $n d 43^{\text {rd }}$ \\
\hline 8 & $H K Y+I+G$ & 690 & $\operatorname{rag} 11^{\text {st }}, c m o s 2^{\text {nd }}, \operatorname{cmos} 1^{\mathrm{s}}$ \\
\hline 9 & $G T R+G$ & 491 & cmos $3^{\text {rd }}, \operatorname{rag} 13^{\text {rd }}$ \\
\hline 10 & $G T R+I+G$ & 367 & cytb $1^{\text {st }}$ \\
\hline 11 & GTR+G & 367 & cytb $3^{\text {rd }}$ \\
\hline
\end{tabular}


Table S3. Partitions and models of sequence evolution used in the Bayesian (BEAST) phylogenetic analyses.

\begin{tabular}{|c|c|c|c|}
\hline Subset & Best Model & \# sites & Partition names \\
\hline 1 & $G T R+1+G$ & 529 & $16 s$ \\
\hline 2 & $\mathrm{~K} 80+\mathrm{G}$ & 233 & $b d n f 1^{\text {st }}$ \\
\hline 3 & $H K Y+I+G$ & 759 & $b d n f 3^{\text {rd }}, b d n f 2^{\text {nd }}, \operatorname{rag} 12^{\text {nd }}$ \\
\hline 4 & GTR+l+G & 600 & cytb $1^{\text {st }}, n d 41^{\text {st }}$ \\
\hline 5 & $\mathrm{GTR}+\mathrm{I}+\mathrm{G}$ & 600 & $n d 42^{\text {nd }}$, cytb $2^{\text {nd }}$ \\
\hline 6 & $\mathrm{GTR}+\mathrm{I}+\mathrm{G}$ & 600 & cytb $3^{\text {rd }}, n d 43^{\text {rd }}$ \\
\hline 7 & $\mathrm{HKY}+\mathrm{G}$ & 690 & $\operatorname{cmos} 1^{\text {st }}, \operatorname{cmos} 2^{\text {nd }}, \operatorname{rag} 11^{\text {st }}$ \\
\hline 8 & $\mathrm{HKY}+\mathrm{G}$ & 491 & $\operatorname{rag} 13^{\text {rd }}$, cmos $3^{\text {rd }}$ \\
\hline
\end{tabular}


Table S4. Node calibration settings used in the BEAST analysis.

\begin{tabular}{|c|c|c|c|c|c|c|}
\hline S.no. & & Node calibrations & Offset & mean & $5 \%$ & $95 \%$ \\
\hline & 1 & Oldest divergence within crown Alethinophidia & 93.9 & 2 & 94.1 & 100.2 \\
\hline & 2 & Oldest divergence between non-xenodermid colubroids and their closest living relative & 50.5 & 6 & 51.2 & 69.35 \\
\hline & 3 & Divergence between Boinae and its sister taxon & 58.0 & 2 & 58.2 & 64.3 \\
\hline & 4 & Divergence between Viperinae and Crotalinae & 20.0 & 1 & 20.1 & 23.1 \\
\hline & 5 & Oldest divergence within elapids & 17.0 & 10 & 18.2 & 48.4 \\
\hline
\end{tabular}


Table S5. Pairwise uncorrected genetic distances of bdnf gene sequences for 92 taxa. Values in bold are within family/subfamily genetic distances.

\begin{tabular}{|c|c|c|c|c|c|c|c|c|c|c|c|c|c|c|c|c|c|c|c|c|c|c|}
\hline & Family (subfamily) & 1 & 2 & 3 & 4 & 5 & 6 & 7 & 8 & 9 & 10 & 11 & 12 & 13 & 14 & 15 & 16 & 17 & 18 & 19 & 20 & 21 \\
\hline 1 & Colubridae (Calamariinae) & & & & & & & & & & & & & & & & & & & & & \\
\hline 2 & Colubridae (Dipsadinae) & $0.01-0.02$ & $0.01-0.02$ & & & & & & & & & & & & & & & & & & & \\
\hline 3 & $\begin{array}{l}\text { Colubridae } \\
\text { (Pseudoxenodontinae) }\end{array}$ & $0.02-0.04$ & $0.01-0.03$ & ${ }^{3} 0-0.04$ & & & & & & & & & & & & & & & & & & \\
\hline 4 & Colubridae (Natricinae) & 0.02 & $0.01-0.02$ & $20.01-0.03$ & & & & & & & & & & & & & & & & & & \\
\hline 5 & Colubridae (Colubrinae) & $0.02-0.04$ & $0.01-0.03$ & $30.01-0.05$ & $0.02-0.03$ & $0-0.03$ & & & & & & & & & & & & & & & & \\
\hline 6 & Colubridae (Grayiinae) & 0.02 & $0.01-0.02$ & $20.02-0.04$ & 0.02 & $0.02-0.03$ & & & & & & & & & & & & & & & & \\
\hline 7 & Elapidae & $0.02-0.03$ & $0.01-0.02$ & $20.02-0.04$ & $0.02-0.03$ & $0.02-0.03$ & $0.02-0.03$ & $0-0.02$ & & & & & & & & & & & & & & \\
\hline 8 & Elapidae (Hydrophiinae) & 0.02 & 0.01 & $0.01-0.03$ & 0.02 & $0.01-0.03$ & 0.02 & 0.01 & & & & & & & & & & & & & & \\
\hline 9 & Elapidae (Laticaudinae) & 0.02 & $0.01-0.02$ & $20.02-0.04$ & 0.02 & $0.02-0.03$ & 0.02 & $0.01-0.02$ & 0 & & & & & & & & & & & & & \\
\hline 10 & Homalopsidae & 0.02 & $0.01-0.02$ & $20.02-0.04$ & 0.02 & $0.02-0.03$ & 0.02 & 0.02 & 0.02 & 0.02 & & & & & & & & & & & & \\
\hline 11 & $\begin{array}{l}\text { Lamprophiidae } \\
\text { (Lamprophiinae) }\end{array}$ & 0.03 & $0.02-0.03$ & $30.03-0.05$ & 0.03 & $0.03-0.04$ & 0.03 & 0.03 & 0.03 & 0.03 & 0.03 & & & & & & & & & & & \\
\hline 12 & $\begin{array}{l}\text { Lamprophiidae } \\
\text { (Aparallactinae) }\end{array}$ & 0.02 & $0.01-0.02$ & $20.01-0.04$ & 0.02 & $0.01-0.03$ & $0.01-0.02$ & $0.01-0.02$ & 0.01 & $0.01-0.02$ & 0.02 & 0.02 & 0.01 & & & & & & & & & \\
\hline 13 & $\begin{array}{l}\text { Lamprophiidae } \\
\text { (Atractaspidinae) }\end{array}$ & 0.02 & $0.01-0.02$ & $20.01-0.03$ & 0.02 & $0.02-0.03$ & 0.02 & $0.01-0.02$ & 0.01 & 0.02 & 0.02 & 0.03 & $0.01-0.02$ & 0.01 & & & & & & & & \\
\hline 14 & $\begin{array}{l}\text { Lamprophiidae } \\
\text { (Psammophiinae) }\end{array}$ & 0.02 & $0.01-0.02$ & $20.02-0.04$ & 0.02 & $0.02-0.03$ & 0.02 & $0.01-0.02$ & 0.01 & 0.02 & 0.02 & 0.03 & 0.02 & 0.02 & & & & & & & & \\
\hline 15 & $\begin{array}{l}\text { Lamprophiidae } \\
\text { (Pseudoxyrhophinae) }\end{array}$ & 0.02 & $0.01-0.02$ & $20.02-0.04$ & 0.02 & $0.02-0.03$ & 0.02 & 0.02 & 0.01 & $0.01-0.02$ & 0.02 & $0.02-0.03$ & $0.01-0.02$ & 0.02 & 0.02 & 0.02 & & & & & & \\
\hline 16 & Pareidae (Pareinae) & $0.03-0.04$ & $0.03-0.04$ & $4 \quad 0.04-0.06$ & 0.04 & $0.03-0.06$ & $0.04-0.05$ & $0.04-0.05$ & 0.04 & 0.04 & $0.04-0.05$ & $0.05-0.06$ & 0.04 & 0.04 & 0.04 & 0.04 & 0.02 & & & & & \\
\hline 17 & $\begin{array}{l}\text { Pareidae (Xylophiinae } \\
\text { subfam. nov.) }\end{array}$ & 0.02 & $0.02-0.03$ & $30.03-0.04$ & 0.02 & $0.03-0.04$ & 0.03 & $0.03-0.04$ & 0.03 & 0.02 & 0.03 & 0.04 & 0.03 & 0.03 & 0.03 & $0.02-0.03$ & $0.02-0.03$ & 0.01 & & & & \\
\hline 18 & Viperidae (Azemiopinae) & 0.02 & $0.01-0.02$ & $20.01-0.03$ & 0.02 & $0.02-0.03$ & 0.02 & 0.02 & 0.01 & 0.02 & 0.02 & 0.03 & $0.01-0.02$ & 0.02 & 0.02 & $0.01-0.02$ & $0.03-0.04$ & 0.02 & & & & \\
\hline 19 & Viperidae (Crotalinae) & 0.02 & $0.01-0.02$ & $20.02-0.04$ & 0.02 & $0.02-0.04$ & 0.02 & $0.02-0.03$ & 0.02 & 0.02 & 0.02 & 0.03 & 0.02 & 0.02 & 0.02 & 0.02 & $0.03-0.04$ & 0.02 & $0-0.01$ & $0-0.01$ & & \\
\hline 20 & Viperidae (Viperinae) & $0.02-0.03$ & 0.02 & $0.02-0.04$ & 0.02 & $0.02-0.04$ & 0.03 & $0.02-0.03$ & 0.02 & 0.02 & $0.02-0.03$ & 0.04 & $0.02-0.03$ & 0.02 & $0.02-0.03$ & $0.02-0.03$ & $0.03-0.04$ & 0.03 & 0.01 & $0.01-0.02$ & 0.01 & \\
\hline 21 & Xenodermidae & $0.04-0.05$ & $0.03-0.05$ & $5 \quad 0.04-0.08$ & $0.04-0.05$ & $0.03-0.07$ & $0.04-0.05$ & $0.04-0.06$ & $0.04-0.05$ & $0.03-0.05$ & $0.04-0.05$ & $0.05-0.06$ & $0.04-0.05$ & $0.04-0.05$ & $0.04-0.05$ & $0.04-0.05$ & $0.05-0.06$ & $0.04-0.05$ & $0.03-0.04$ & 4 $0.03-0.04$ & $0.03-0.05$ & 50.03 \\
\hline
\end{tabular}


Table S6. Pairwise uncorrected genetic distances of cmos gene sequences for 308 taxa. Values in bold are within family/subfamily genetic distances.

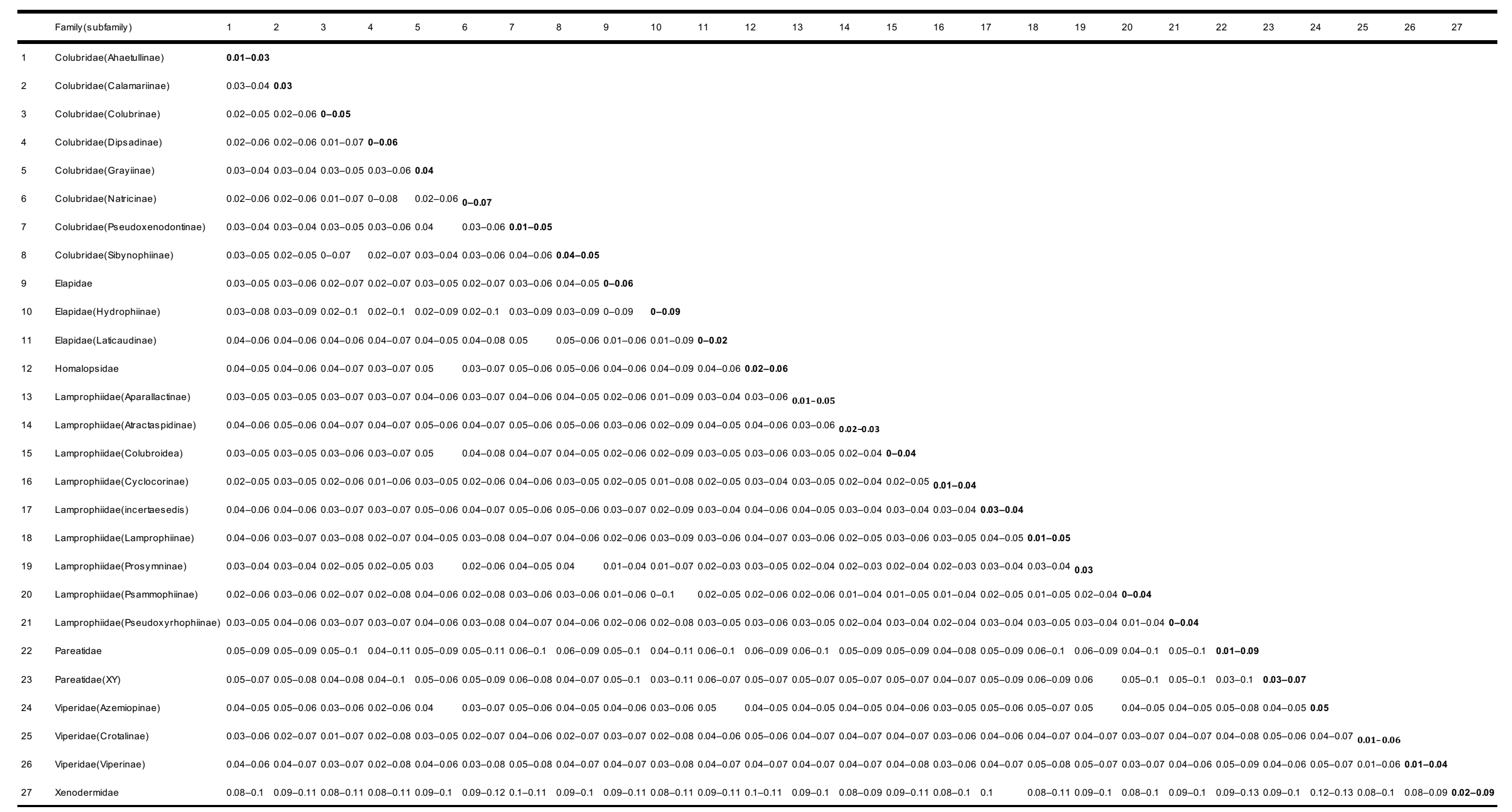


Table S7. Pairwise Kimura 2-parameter genetic distances of bdnf gene sequences for 92 taxa. Values in bold are within family/subfamily genetic distances.

\begin{tabular}{|c|c|c|c|c|c|c|c|c|c|c|c|c|c|c|c|c|c|c|c|c|c|c|}
\hline & Family (subfamily) & 1 & 2 & 3 & 4 & 5 & 6 & 7 & 8 & 9 & 10 & 11 & 12 & 13 & 14 & 15 & 16 & 17 & 18 & 19 & 20 & 21 \\
\hline 1 & Colubridae (Calamariinae) & & & & & & & & & & & & & & & & & & & & & \\
\hline 2 & Colubridae (Dipsadinae) & $0.01-0.02$ & $0.01-0.02$ & & & & & & & & & & & & & & & & & & & \\
\hline 3 & $\begin{array}{l}\text { Colubridae } \\
\text { (Pseudoxenodontinae) }\end{array}$ & $0.02-0.04$ & $0.01-0.03$ & $30-0.04$ & & & & & & & & & & & & & & & & & & \\
\hline 4 & Colubridae (Natricinae) & 0.02 & $0.01-0.02$ & $0.01-0.03$ & & & & & & & & & & & & & & & & & & \\
\hline 5 & Colubridae (Colubrinae) & $0.02-0.04$ & $0.01-0.03$ & $0.01-0.05$ & $0.02-0.03$ & $0-0.03$ & & & & & & & & & & & & & & & & \\
\hline 6 & Colubridae (Grayiinae) & 0.02 & $0.01-0.02$ & $0.02-0.04$ & 0.02 & $0.02-0.03$ & & & & & & & & & & & & & & & & \\
\hline 7 & Elapidae & $0.02-0.03$ & $0.01-0.02$ & $0.02-0.05$ & $0.02-0.03$ & $0.02-0.04$ & $0.02-0.03$ & $0-0.02$ & & & & & & & & & & & & & & \\
\hline 8 & Elapidae (Hydrophiinae) & 0.02 & 0.01 & $0.01-0.03$ & 0.02 & $0.01-0.03$ & 0.02 & 0.01 & & & & & & & & & & & & & & \\
\hline 9 & Elapidae (Laticaudinae) & 0.02 & $0.01-0.02$ & $0.02-0.04$ & 0.02 & $0.02-0.03$ & 0.02 & $0.01-0.02$ & 0.01 & & & & & & & & & & & & & \\
\hline 10 & Homalopsidae & 0.02 & $0.01-0.02$ & $0.02-0.04$ & 0.02 & $0.02-0.03$ & 0.03 & 0.02 & 0.02 & 0.02 & & & & & & & & & & & & \\
\hline 11 & Lamprophiidae (Lamprophiinae) & 0.03 & $0.02-0.03$ & $0.03-0.05$ & 0.03 & $0.03-0.04$ & 0.03 & 0.03 & 0.03 & 0.03 & 0.03 & & & & & & & & & & & \\
\hline 12 & Lamprophiidae (Aparallactinae) & 0.02 & $0.01-0.02$ & $0.01-0.04$ & 0.02 & $0.01-0.03$ & $0.01-0.02$ & $0.01-0.02$ & 0.01 & $0.01-0.02$ & 0.02 & 0.02 & 0.01 & & & & & & & & & \\
\hline 13 & Lamprophiidae (Atractaspidinae) & 0.02 & $0.01-0.02$ & $20.01-0.04$ & 0.02 & $0.02-0.03$ & 0.02 & $0.01-0.02$ & 0.01 & 0.02 & 0.02 & 0.03 & $0.01-0.02$ & 0.01 & & & & & & & & \\
\hline 14 & Lamprophiidae (Psammophiinae) & 0.02 & $0.01-0.02$ & $0.02-0.04$ & 0.02 & $0.02-0.04$ & 0.02 & $0.01-0.02$ & 0.01 & 0.02 & 0.02 & 0.03 & 0.02 & 0.02 & & & & & & & & \\
\hline 15 & $\begin{array}{l}\text { Lamprophiidae } \\
\text { (Pseudoxyrhophiinae) }\end{array}$ & 0.02 & $0.01-0.02$ & $0.02-0.04$ & 0.02 & $0.02-0.03$ & 0.02 & 0.02 & 0.01 & $0.01-0.02$ & 0.02 & $0.02-0.03$ & $0.01-0.02$ & 0.02 & 0.02 & $0.01-0.02$ & & & & & & \\
\hline 16 & Pareidae (Pareinae) & $0.03-0.04$ & $0.03-0.05$ & $50.04-0.07$ & 0.04 & $0.04-0.06$ & $0.04-0.05$ & $0.04-0.05$ & 0.04 & 0.04 & $0.04-0.05$ & $0.05-0.06$ & $0.04-0.05$ & $0.04-0.05$ & $0.04-0.05$ & $0.04-0.05$ & 0.02 & & & & & \\
\hline 17 & $\begin{array}{l}\text { Pareidae (Xylophinae subfam. } \\
\text { nov.) }\end{array}$ & 0.02 & $0.02-0.03$ & $30.03-0.05$ & 0.02 & $0.03-0.05$ & 0.03 & $0.03-0.04$ & 0.03 & 0.02 & 0.03 & 0.04 & 0.03 & 0.03 & 0.03 & 0.03 & $0.02-0.03$ & & & & & \\
\hline 18 & Viperidae (Azemiopinae) & 0.02 & $0.01-0.02$ & $0.01-0.04$ & 0.02 & $0.02-0.03$ & 0.02 & 0.02 & 0.01 & 0.02 & 0.02 & 0.03 & $0.01-0.02$ & 0.02 & 0.02 & $0.01-0.02$ & $0.03-0.04$ & 0.02 & & & & \\
\hline 19 & Viperidae (Crotalinae) & 0.02 & $0.01-0.02$ & $0.02-0.04$ & 0.02 & $0.02-0.04$ & $0.02-0.03$ & $0.02-0.03$ & 0.02 & 0.02 & 0.02 & $0.03-0.04$ & 0.02 & 0.02 & $0.02-0.03$ & 0.02 & $0.03-0.04$ & 0.02 & $0-0.01$ & $0-0.01$ & & \\
\hline 20 & Viperidae (Viperinae) & 0.03 & 0.02 & $0.02-0.04$ & 0.02 & $0.02-0.04$ & 0.03 & $0.02-0.03$ & 0.02 & 0.02 & $0.02-0.03$ & 0.04 & $0.02-0.03$ & 0.02 & 0.03 & $0.02-0.03$ & $0.03-0.05$ & 0.03 & 0.01 & $0.01-0.02$ & 0.01 & \\
\hline 21 & Xenodermidae & $0.04-0.06$ & $0.03-0.05$ & $0.04-0.08$ & $0.04-0.06$ & $0.03-0.07$ & $0.04-0.06$ & $0.04-0.06$ & $0.04-0.05$ & $0.04-0.05$ & $0.04-0.05$ & $0.05-0.07$ & $0.04-0.06$ & $0.04-0.06$ & $0.04-0.05$ & $0.04-0.06$ & $0.05-0.07$ & $0.04-0.05$ & $50.03-0.04$ & $0.03-0.05$ & $0.04-0.05$ & 50.03 \\
\hline
\end{tabular}


Table S8. Pairwise Kimura 2-parameter genetic distances of cmos gene sequences for 92 taxa. Values in bold are within family/subfamily genetic distances.

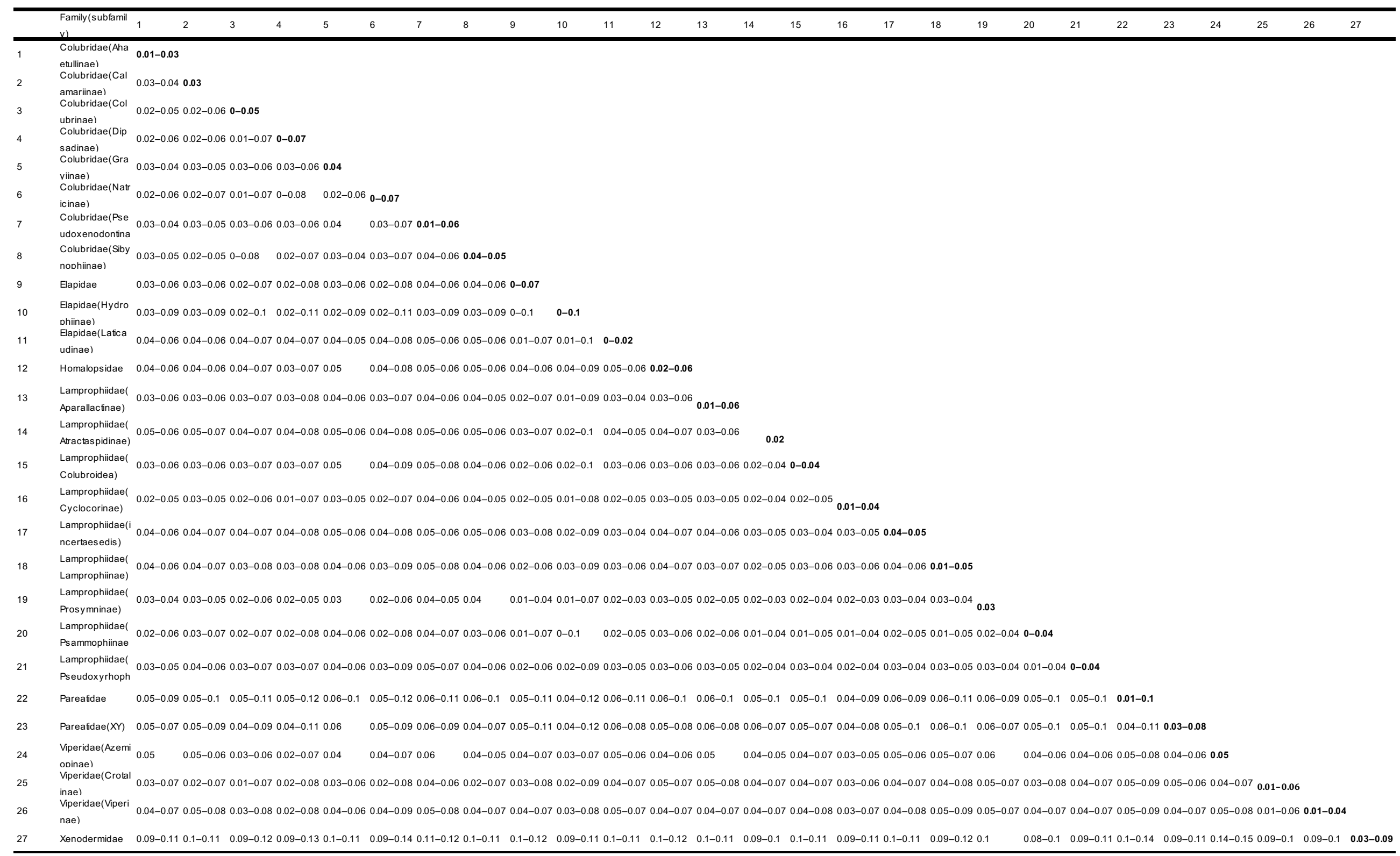

